\title{
Hypoxia-induced IncRNA -NEAT1 sustains the growth of hepatocellular carcinoma via regulation of miR-199a-3p/UCK2: integrative bioinformatics analysis and experimental validation
}

Qiangnu Zhang

Shenzhen People's Hospital

Qian Cheng

West China School of Public Health and West China Fourth Hospital

Mengting Xia

North Sichuan Medical College

Xiaotao Huang

North Sichuan Medical College

Xiaoyan He

The fifth affilated hospital of Zhengzhou University

Juan Liao ( $\square$ juanliao@scu.edu.cn)

West China School of Public Health and West China Fourth Hospital https://orcid.org/0000-00020386-1506

\section{Research}

Keywords: Hepatocellular carcinoma, LncRNA-NEAT1, microRNA, UCK2, ceRNA, Hypoxia

Posted Date: March 18th, 2020

DOI: https://doi.org/10.21203/rs.3.rs-17617/v1

License: (c) (i) This work is licensed under a Creative Commons Attribution 4.0 International License. Read Full License 


\section{Abstract}

Background: The long noncoding RNA (IncRNA) nuclear paraspeckle assembly transcript 1 (NEAT1) has emerged as a novel player in hepatocellular carcinoma (HCC). Hypoxia is a common characteristic of the microenvironment of HCC. However, it remains unclear whether IncRNA-NEAT1 is induced by hypoxia in HCC, and the mechanism that underlies LncRNA-NEAT1 function is not well characterized.

Methods: The expression changes of IncRNA-NEAT1 in HCC cell lines under hypoxic conditions were examined by quantitative reverse transcription-polymerase chain reaction (qRT-PCR). The regulatory effect of HIF-1a on IncRNA-NEAT1 was confirmed with chromatin immunoprecipitation (ChIP) and luciferase reporter assays. The function of IncRNA-NEAT1 on HCC cell growth under hypoxic conditions was determined by CCK-8 assay and flow cytometry. IncRNA -NEAT1 was predicted to serve as a competing endogenous RNA (ceRNA) within microRNA (miRNA)/mRNA axes based on microarray data, public HCC-related datasets and integrative bioinformatics analysis, and the miR-199a-3p/UCK2 axis was selected and validated by qRT-PCR, western blotting, RNA immunoprecipitation, and luciferase reporter analyses. The role of miR-199a-3p/UCK2 in HCC and its functional association with IncRNA-NEAT1 were assessed both in vitro and in vivo .

Results : LncRNA-NEAT1 expression was significantly induced by hypoxia in SNU-182 and HUH7 cells. HIF-1a was shown to regulate IncRNA-NEAT1 transcription. Under hypoxic conditions, IncRNA-NEAT1 maintained the growth of HCC cells and inhibited apoptosis and cell cycle arrest. LncRNA-NEAT1 was predicted to regulate a panel of HCC-associated miRNA-mRNA pairs consisting of 8 miRNAs and 13 mRNAs. Furthermore, IncRNA-NEAT1 was demonstrated to serve as a miR-199a-3p sponge that regulates UCK2 expression and to play a tumor-suppressive role in HCC, while UCK2 promotes cell growth. LncRNANEAT1 was shown to function as a ceRNA of miR-199a-3p/UCK2 both in HCC cells under hypoxic conditions and in an animal model.

Conclusion: LncRNA-NEAT1 is a hypoxia-responsive IncRNA in HCC that sustains the growth of HCC cells by regulating HCC-associated mRNAs that interact with tumor-suppressive miRNAs. The IncRNANEAT1/miR-199a-3p/UCK2 pathway may contribute to the progression of HCC in a hypoxic microenvironment and therefore may represent a novel therapeutic target for HCC.

\section{Background}

Hepatocellular carcinoma (HCC) is one of the most commonly diagnosed primary cancers of the liver and the leading cause of cancer-related mortality worldwide, accounting for more than 780,000 deaths in 2018[1]. Although progress has been made in recent years in diagnostic, treatment, and screening technologies, HCC is often diagnosed at advanced stages, and in most cases, advanced HCC patients miss the best surgical window[2]. Unfortunately, the effects of chemoradiotherapy, targeted therapy, and immunotherapy against HCC are rather limited, with dismal survival rates for most HCC patients[3]. Therefore, there is an urgent need to identify novel targets and molecular markers for the diagnosis and 
treatment of HCC. Concerted efforts are needed to better understand the pathophysiological mechanisms underlying the development and progression of HCC.

Abnormal vascular networks surrounding solid tumors and the requirement of excess oxygen for rapid growth of cancer cells can lead to hypoxia, which is a common characteristic of the microenvironment of solid tumors[4]. At the cellular level, hypoxia induces angiopoiesis, metabolic reprogramming, epithelialmesenchymal transition, remodeling of extracellular mechanisms, stemness, and immune escape. Clinically, hypoxia contributes to the aggressive clinical characteristics of HCC and resistance to both radiotherapy and chemotherapy $[5,6]$. Genes, especially those that are regulated by hypoxia inducible factors (HIFs), as well as signal transduction related to the hypoxic microenvironment, have been a common focus over the last decade. Nevertheless, it is still a challenge to treat HCC by targeting hypoxia.

Considerable evidence suggests that hypoxia regulates long non-coding RNA (IncRNAs) that involved in the onset and progression of various cancers. Hypoxia responsive IncRNAs may play clinical roles on patients' progression and prognosis by regulating proliferation, migration, invasion, and therapy resistance of cancer cells[7, 8]. For instance, Deng et al. found that IncRNA-BX111887 transcription is induced by HIF-1a in response to hypoxia, which enhances the proliferation and invasion of pancreatic cancer cells[9]. LncRNA-MALAT1 expression is also dramatically increased in HCC cells in response to hypoxic conditions, whereas knock-down of MALAT1 counteracts the tumor-promoting effect of hypoxia[10]. Thus, elucidation of the roles of IncRNAs under hypoxic conditions is crucial to better understand the onset, features, and poor clinical outcome of HCC.

The IncRNA nuclear paraspeckle assembly transcript 1 (NEAT1) has been reported as a novel player in the onset and progression of HCC. Overexpression of NEAT1 drives HCC progression and has been correlated with the poor prognosis of patients[11, 12]. Furthermore, Choudhry et al. demonstrated that IncRNA-NEAT1 activation in response to hypoxia promotes the survival of breast cancer cells[13]. However, the activation of IncRNA-NEAT1 in response to hypoxia has not been elucidated in HCC, and IncRNA-NEAT1-regulated downstream pathways are not well established. Therefore, in the present study, we investigated the response of IncRNA-NEAT1 to hypoxia and revealed its mechanism, which involves transcriptional regulation by HIF-1a. Potential miRNA and mRNA targets of IncRNA-NEAT1 were selected and filtered by an integrative bioinformatics analysis approach that was based on numerous HCC-related datasets. Moreover, candidate miRNA and mRNA targets were validated in vitro and in vivo.

\section{Materials And Methods}

\section{Cell culture and hypoxic conditions}

HCC cells (SNU-182 and HUH7) were obtained from the American Type Culture Collection (Manassas, VA, USA) and routinely cultured in Roswell Park Memorial Institute 1640 (for SNU-182 cells) or Dulbecco's Modified Eagle's Medium (for HUH7 cells) supplemented with $10 \%$ fetal bovine serum, $100 \mathrm{U} / \mathrm{mL}$ of penicillin, and $100 \mathrm{mg} / \mathrm{mL}$ of streptomycin (Hyclone Laboratories, Inc., South Logan, UT, USA) under an 
atmosphere of $5 \% \mathrm{CO}_{2}$ at $37^{\circ} \mathrm{C}$. As a model for hypoxia, SNU-182 and $\mathrm{HUH7}$ cells were cultured under an atmosphere of $1 \% \mathrm{O}_{2} / 5 \% \mathrm{CO}_{2} / 94 \% \mathrm{~N}_{2}$ for $24 \mathrm{~h}$ and then simulated with $\mathrm{CoCl}_{2}(100 \mu \mathrm{M})$, a hypoxia mimetic agent, for $24 \mathrm{~h}$.

\section{Cell transfection}

Cells were transfected with the plasmid pcDNA3.1-NEAT1 to up-regulate IncRNA-NEAT1 expression, while the plasmid pcDNA3.1-UCK2 was used to overexpress UCK2. The empty plasmid pcDNA3.1 served as a negative transfection control. Small interfering RNA for IncRNA-NEAT1 (siRNA-NEAT1) UCK2 (siRNAUCK2) and HIF-1 a (siRNA- HIF-1a) were used to silence their expression (Genepharma, Shanghai, China). To upregulate candidate miRNAs, miR-mimics were obtained. AllStars Negative Control siRNA was used to transfect cells with siRNA and miR-mimics (Qiagen, Hilden, Germany). Prior to experimentation, the cells were transfected for 24 or $48 \mathrm{~h}$. To obtain stably transfected SNU-182 cells, lentiviral vectors were prepared by Genechem Company (Shanghai, China) and used to deliver IncRNA-NEAT1 (Lv-NEAT1), miR199a-3p (Lv-miR-199a-3p), and shRNA-UCK2 (Lv-shRNA-UCK2). Successful transfection was confirmed by quantitative reverse transcription-polymerase chain reaction (qRT-PCR) analysis after $48 \mathrm{~h}$ (Additional file 1: Supplementary Fig. 1).

\section{qRT-PCR analysis}

Total RNA was isolated using TRIzol reagent (Life Technologies Corporation, Carlsbad, CA, USA), quantified, and reverse transcribed into complementary DNA (cDNA) using PrimeScript ${ }^{\text {TM }}$ RT Master Mix and the PrimeScript ${ }^{\text {TM }}$ RT Reagent Kit (Takara Bio, Inc., Shiga, Japan). Then, the cDNA samples were analyzed using the SYBR ${ }^{\circledR}$ Premix Ex Taq ${ }^{\text {TM }}$ II Kit (Takara Bio, Inc., Shiga, Japan) with glyceraldehyde 3phosphate dehydrogenase (GAPDH) as an internal control. For miRNA analysis, cDNAs were generated and amplified using the Mir- ${ }^{\mathrm{TM}}$ miRNA First-Strand Synthesis and qRT-PCR TB Green® Kit (Takara Bio, Inc., Shiga, Japan). U6 was used as reference for miRNA analysis. The PCR protocol was conducted in accordance with the manufacturer's instructions using the primers shown in Additional file 1: Supplementary Table S1.

\section{Western blot analysis}

Total protein samples were prepared in radioimmunoprecipitation assay buffer (Beyotime Institute of Biotechnology, Shanghai, China) containing protease inhibitor cocktail. Samples with equal amounts of protein were loaded into the wells of a $12 \%$ polyacrylamide gel and separated by electrophoresis. Then, the protein bands were transferred onto nitrocellulose blotting membranes, which were blocked with $5 \%$ fat-free milk and incubated with primary antibodies (Abcam, Cambridge, UK) against UCK2 (dilution, 1:1000) and GAPDH (1:2000) for $12 \mathrm{~h}$ at $4^{\circ} \mathrm{C}$. After incubation with IRDye ${ }^{\circledR}$ secondary antibody (LI-COR Biosciences, Lincoln, NE, USA), the protein bands were imaged using the Odyssey $\circledast$ Infrared Imaging System (LI-COR Biosciences).

\section{Chromatin immunoprecipitation (ChIP) analysis}


ChIP analysis was conducted using the ab500 ChIP Assay KIT with anti-HIF-1a antibody (Abcam, Cambridge, UK) in accordance with the manufacturer's instructions. Immunoglobulin G (IgG) served as a control. The resulting DNA fragments were amplified by PCR with the primers listed in Additional file 1: Supplementary Table S1. The PCR products were analyzed by electrophoresis.

\section{RNA immunoprecipitation (RIP) assay}

RIP analysis was performed using the Imprint ${ }^{\circledR}$ RNA Immunoprecipitation Kit (Sigma-Aldrich Corporation, St. Louis, MO, USA) in accordance with the manufacturer's instructions, with an antibody against argonaute-2 (AGO2, Abcam, Cambridge, UK). IgG served as a control. The purified RNAs were then subjected to qRT-PCR analysis.

\section{Luciferase reporter assay}

The 3' untranslated region of the wild-type (WT) or mutant IncRNA-NEAT1 (or UCK2) sequence was inserted into the psiCHECK-2 luciferase reporter vector (Promega Corporation, Madison, WI, USA). After $48 \mathrm{~h}$ of co-transfection with the luciferase reporter vector and miR-199a-3p mimic (or AllStars Negative Control), the cells were lysed, and luciferase activity was measured using the Dual-Luciferase ${ }^{\circledR}$ Reporter Assay System (Promega Corporation). Renilla luciferase activity was normalized to firefly luciferase activity. SNU-182 cells or HIF-1a knock-down cells were transfected with luciferase reporter vectors containing the WT or mutant putative hypoxia response element (HRE) sequence (ACGTGC) and then treated with $\mathrm{CoCl}_{2}$ for $24 \mathrm{~h}$. A similar luciferase reporter assay was performed to assess the effect of HIF1a on the promoter of IncRNA-NEAT1.

\section{Proliferation analysis}

Cell proliferation was assessed using the Cell Counting Kit-8 (CCK-8; Sigma-Aldrich Corporation). In brief, $1 \times 10^{4}$ cells were seeded into the wells of 96 -well plates and cultured for $24 \mathrm{~h}$. Then, adherent cells were cultured under normoxic or hypoxic conditions. After 24,48 , or 72 h of culture, the cells were incubated with $10 \% \mathrm{CCK}-8$ reagent at $37^{\circ} \mathrm{C}$ for $1 \mathrm{~h}$. Cell viability was determined by measuring the absorbency at $450 \mathrm{~nm}$. The relative proliferation rate was calculated as the cell viability at 24,48, or $72 \mathrm{~h} /$ cell viability at $0 \mathrm{~h}$. The viability of untreated adherent cells was assessed at $0 \mathrm{~h}$.

\section{Flow cytometry analysis}

At $48 \mathrm{~h}$ after transfection or $24 \mathrm{~h}$ under hypoxic conditions, the cells were harvested and washed with phosphate-buffered saline. The proportion of apoptotic cells was determined by flow cytometry with an Annexin V-FITC Apoptosis Detection Kit (Beyotime Institute of Biotechnology, Shanghai, China). For cell cycle analysis, harvested cells were fixed with $70 \%$ cold ethanol for $12 \mathrm{~h}$ and then treated with propidium iodide for $30 \mathrm{~min}$. The proportion of apoptotic cells and the cell cycle distribution were measured by flow cytometry using a FACSCalibur ${ }^{\mathrm{TM}}$ Flow Cytometer (BD Biosciences, San Jose, CA, USA). Data were analyzed using the FlowJo ${ }^{\mathrm{TM}}$ platform for flow cytometry analysis (version 10; FlowJo LLC, Ashland, OR, USA).

\section{Microarray analysis}


SNU-182 cells were transfected with pcDNA3.1-NEAT1. After 48 h, RNA was collected and analyzed by Agilent Whole human genome Microarray and Human miRNA Microarray, Release 21.0 (Agilent Technologies, Santa Clara, CA, USA). The differentially expressed mRNAs and microRNAs (fold change > 1.5 and $\mathrm{P}<0.05$, control vs. pcDNA3.1-NEAT1 transfection) were identified using $\mathrm{R}$ with Limma package.

\section{Animal tumor model}

Twelve BALB/c nude mice, aged 4-6 weeks, were purchased from Biolite Biological Engineering Co., Ltd. (Nanjing, China) and subcutaneously injected with SNU-182 cells stably transfected with Lv-NEAT1, LvmiR-199a-3p, Lv- shRNA-UCK2, or Lv-control ( $5 \times 10^{6}$ cells/mouse, $\mathrm{n}=3$ for each group). Tumor diameters were measured every 3 days. The tumor volumes were calculated as $0.5 \times\left(\right.$ length $\times$ width $\left.^{2}\right)$. All mice were sacrificed on day 24 , and the tumors were resected and weighed. All animal experiments were performed in accordance with the guidelines of the Research Animal Care Committee of Zhengzhou University (Zhengzhou, Henan, China).

\section{Statistical analysis}

Statistical analysis was performed with R software (version 3.5.3; https://www.r-project.org/). Normally distributed data are presented as the mean \pm standard deviation. Non-normally distributed data are presented as median values. The t-test was used to identify significant differences between two sets of normally distributed data, while one-way analysis of variance was used identify differences among multiple groups. Non-normally distributed data were analyzed using the Mann-Whitney $U$ test. The significance of survival data was determined using the log-rank test. A probability ( $p)$ value of $<0.05$ was considered statistically significant.

\section{Bioinformatics analysis}

Liver cancer transcriptome profiling data were downloaded from The Cancer Genome Atlas (TCGA) database (https://www.cancergenome.nih.gov). The expression profile data of one miRNA (GSE36915) and 7 mRNAs (GSE14520, GSE22058, GSE25097, GSE36376, GSE45436, GSE64041, and GSE76427) of HCC patients were retrieved from the Gene Expression Omnibus database https://www.ncbi.nlm.nih.gov/geo/). Differentially expressed genes and miRNAs were extracted from each dataset with the Limma package of R software (version 3.5.3). The RobustRankAggreg package of $R$ was used for integrated analysis of the expression profile data of the $7 \mathrm{mRNAs}$. Survival analysis was performed using the "survival" package, and data visualization was performed using the "ggplot2" package of R. The open-source online software (ENCORI v1.0) provided by Encyclopedia of RNA Interactome database (http://starbase.sysu.edu.cn/index.php) was used to predict IncRNANEAT1/miRNA interactions. miRWalk 2.0 online software (http://zmf.umm.uniheidelberg.de/apps/zmf/mirwalk2/) and TargetScan web server (http://www.targetscan.org/vert_72/) were used to predict miRNA-target interactions and the binding sites of candidate miRNAs and mRNAs. Gene ontology enrichment analysis were performed using online tools provided by DAVID Bioinformatics Resources 6.8 (https://david.ncifcrf.gov/). microRNA pathway analyses were conducted using mirPath v.3(http://snf-515788.vm.okeanos.grnet.gr/). 


\section{Results}

\section{LncRNA-NEAT1 is induced by hypoxia via HIF-1a in HCC cells}

To evaluate the response of IncRNA-NEAT1 to hypoxia, we cultured SNU-182 and HUH7 cells under hypoxic conditions $\left(1 \% \mathrm{O}_{2}\right)$ or treated them with the hypoxia mimetic agent $\mathrm{CoCl}_{2}$. As shown in Fig. 1a and $b$, both hypoxic conditions and $\mathrm{CoCl}_{2}$ significantly increased IncRNA-NEAT1 expression in SNU-182 cells; however, knock-down of HIF-1 a suppressed the response of IncRNA-NEAT1 to hypoxia. Additionally, inspection of the TCGA-LIHC database revealed that HIF-1 a expression positively correlates with the IncNEAT1 level in HCC tissues (Fig. 1C). Therefore, we speculated that transcription of IncRNA-NEAT1 might be regulated by HIF-1a. Inspection of the genomic sequence showed that there is an HRE (ACGTGC) in the IncRNA-NEAT1 3' upstream region that is predicted to bind HIF-1a (Fig. 1d, left). We further validated the binding of HIF-1a to the IncRNA-NEAT1 promoter by ChIP assay in SNU-182 and HUH7 cells (Fig. 1d, right). Additionally, dual-luciferase reporter assays verified that $\mathrm{CoCl}_{2}$ increases the luciferase activity in cells transfected with plasmids containing the WT HRE sequence, but not a mutant sequence (Fig. 1e). On the other hand, knock-down of HIF-1a reduced the luciferase activity in $\mathrm{CoCl}_{2}$-treated cells for HRE-WT but not HRE-MUT reporter plasmids (Fig. 1f). These data indicate that transcriptional upregulation of IncRNA-NEAT1 in HCC cells under hypoxic conditions is mediated by HIF-1a.

\section{LncRNA-NEAT1 sustains the growth of HCC cells under hypoxic conditions}

To assess the function of IncRNA-NEAT1 in HCC cells in under hypoxic conditions, we evaluated the effect of IncRNA-NEAT1 overexpression and knock-down. Overexpression of IncRNA-NEAT1 had no effect on the viability of SNU-182 cells under normoxic conditions; however, knock-down of IncRNA-NEAT1 inhibited cell viability (Fig. 2a, left panel). Furthermore, under hypoxic conditions, cells transfected with pcDNA3.1-NEAT1 had higher proliferation rates, while knock-down of IncRNA-NEAT1 inhibited proliferation (Fig. 2a, right panel). Consistently, knock-down of IncRNA-NEAT1 induced apoptosis of SNU182 cells, while after $24 \mathrm{~h}$ of exposure to hypoxic conditions, siRNA-NEAT1 treatment increased the proportion of apoptotic cells, which was reduced by IncRNA-NEAT1 overexpression (Fig. 2b). Similarly, overexpression of IncRNA-NEAT1 had no effect on the cell cycle under normoxic conditions. However, IncRNA-NEAT1 knock-down induced G1 arrest after hypoxia treatment for $24 \mathrm{~h}$, and overexpression of IncRNA-NEAT1 antagonized this effect (Fig. 2c). These results were confirmed in HUH7 cells (Additional file 1: Supplementary Fig. 2). Therefore, these findings suggest that Inc-NEAT1 increases the proliferation rate in HCC cells under hypoxic conditions by decreasing the apoptotic rate and promoting G1 arrest.

\section{Identification of a panel of HCC-associated miRNAs and mRNAs that IncRNA-NEAT1 may regulate as competing}




\section{endogenous RNAs (ceRNAs)}

Next, we investigated the mechanisms underlying the effects of IncRNA-NEAT1 in inducing cell growth under hypoxic conditions. LncRNAs are known to regulate mRNAs by sponging miRNAs as ceRNAs. Firstly, we used microarray analysis to identify the differentially expressed microRNAs and mRNAs (>1.5fold with $\mathrm{P}<0.05$, compared with negative transfection control) after pcDNA3.1-NEAT1 transfection in SNU-182 cells. 63 microRNA were significantly down-regulated and 414 mRNA significantly up-regulated after IncRNA-NEAT1 overexpression (Additional file 1: Supplementary Tables 2 and 3). Next, we built a ceRNA network of potential IncRNA-NEAT1/microRNAs/mRNAs combinations based on the microarray data and bioinformatics predictions (see Additional file 1: Supplementary Fig. 3 for workflow). CLIP-Seq analysis data derived from online software provided by Encyclopedia of RNA Interactome database were used to identify miRNAs from our microarray analysis containing 7-mer or 8-mer seed matches that are predicted to bind to IncRNA-NEAT1. To further select HCC-associated miRNAs, the miRNAs were filtered with the use of the following criteria: (a) down-regulation by more than 1.5 -fold $(P<0.05)$ in HCC tissues according to the GSE36915 dataset; and (b) reported in the literature as cancer suppressors in HCC. Eight candidate miRNAs conforming to these criteria were identified (miR-144-5p, miR-129-5p, miR-199a-3p, miR-214-5p, miR-483-3p, miR-486-5p, miR-542-3p, and miR-582-5p). Next, the targeted mRNAs of these 8 candidate miRNAs were predicted using integrated data from TargetScan and miRWalk 2.0. In total, 780 genes were predicted as target mRNAs of the 8 candidate miRNAs. We compared this list of 780 genes with the set of differentially expressed mRNAs measured in pcDNA3.1-NEAT1 transfected cells. To identify HCC-related genes in the overlapping mRNA list, results were further filtered using the expression profiles results obtained from 7 independent HCC-related public datasets (GSE14520, GSE22058, GSE25097, GSE36376, GSE45436, GSE64041, and GSE76427). An integrated list of differentially expressed genes (HCC-RRA-list) was obtained using the robust rank aggregation (RRA) algorithm with genes that were significantly up- or down-regulated $(>1.2$-fold with $\mathrm{P}<0.05$, tumor tissue vs. non-tumor tissue) in all 7 HCC-related datasets. Thirteen genes in the overlapping mRNA list were also in the HCCRRA-list and were up-regulated in HCC tissues. Finally, a potential HCC-associated IncRNA-miRNA-mRNA regulatory flow network was assembled, comprised of IncRNA-NEAT1, 8 candidate miRNAs, and 13 candidate genes (Fig. 3a). To better understand the potential biological function of the candidate miRNAs and mRNAs, functional enrichment analysis based on Gene Ontology (GO) and KEGG (Kyoto Encyclopedia of Genes and Genomes) pathway databases was performed. As shown in Fig. 3b, the 8 candidate miRNAs were mainly associated with the typical tumor-associated pathways (e.g. cell cycle, p53 signaling pathway, pathway in cancers). The enriched GO-biological process terms, -molecular function terms and -cellular component terms are shown in Fig. 3c. Among them, potential terms (e.g. "regulation of cell growth", "positive regulation of cell cycle", and "cellular response to starvation") further support a role for these miRNAs in tumor-related functions.

To further verify the potential role of the 8 miRNAs and 13 mRNAs in HCC, we evaluated their expression patterns reported in public databases. The fold changes (tumor tissue vs. non-tumor tissue) of the 8 candidate miRNAs in the GSE36915 and The Cancer Genome Atlas Liver Hepatocellular Carcinoma 
(TCGA-LIHC) datasets are shown in Fig. 4a. Except for miR-129-5p, which was not included in the TCGA$\mathrm{LIHC}$ dataset, all of the other candidate miRNAs were consistently down-regulated in HCC tissues in the GSE36915 and TCGA-LIHC datasets. Furthermore, all 13 genes were up-regulated in HCC tumor tissues compared with normal tissue $(>1.5$-fold with $\mathrm{P}<0.05)$, as confirmed in the TCGA-LIHC dataset (Fig. $4 \mathrm{~b}$ ). The integrative fold changes of the 13 candidate genes among the 7 HCC-related public datasets are shown in Fig. 4c (tumor tissue vs. non-tumor tissue). We further evaluated the potential roles of these genes in HCC prognosis. In the GSE14520 dataset, 7 of 13 candidate genes (UCK2, LRCC1, GINS2, CDK4, LPGAT1, UBR5, and DLK1) are risk factors for poor survival, while according to the TCGA-LIHC dataset, high expression of 7 of the 13 candidate genes (UCK2, LRRC1, TTF2, GINS2, CDK4, CPD, and SESTD1) predict poor survival of HCC patients (Fig. 4d). These data indicate that InCRNA-NEAT1 may play a role in the progression of HCC through a panel of HCC-related miRNAs and mRNAs associated with HCC.

\section{LncRNA-NEAT1 regulates UCK2 by sponging miR-199a-3p}

Next, we sought to validate the predicted candidate IncRNA-NEAT1-miRNA-mRNA regulatory patterns. Among the 4 genes (UCK2, LRCC1, GINS2, and CDK4) that were identified to be associated with poor survival of HCC patients in both the GSE14520 and TCGA-LIHC datasets, UCK2 had the greatest number of predicted interactions with NEAT1-targeted miRNAs (miR-199a-3p, miR-483-3p, miR-486-5p, miR-582$5 p$, and miR-129-5p) and, thus, can be regarded as a potential hub mediator. Furthermore, UCK2 was found to be an impressive risk factor for survival (hazard ratio $=2.2, P<0.01$ in the GSE14520 dataset; hazard ratio $=2.1, P<0.01$ in the TCGA-LIHC dataset). Therefore, we sought to further validate the functional interaction of UCK2, along with miR-199a-3p, miR-483-3p, miR-486-5p, miR-582-5p, and miR129-5p, with IncRNA-NEAT1. As shown in Fig. 5a, only miR-199a-3p mimic influenced UCK2 mRNA expression in SNU-182 and HUH7 cells. The inhibitory effect of miR-199a-3p on UCK2 was confirmed by western blotting (Fig. 5b). Furthermore, overexpression of IncRNA-NEAT1 was found to suppress the expression of miR-199a-3p (Fig. 5c) and enhance UCK2 mRNA and protein expression (Fig. 5d and e), while IncRNA-NEAT1 knock-down had the opposite effect. The role of miR-199a-3p in mediating the effect of IncRNA-NEAT1 on UCK2 expression was further verified by luciferase reporter assay results, which demonstrated that miR-199a-3p decreases luciferase activity in cells transfected with reporter plasmids containing either the NEAT1-WT or UCK2-WT sequences that are predicted to be binding sites for miR199a-3p, but not corresponding mutant sequences (Fig. $5 f$ and g). In addition, the results of RIP assays demonstrated that IncRNA-NEAT1 and miR-199a-3p were both contained in complexes that were pulled down with AGO2 antibody (Fig. 5h), which suggests that IncRNA-NEAT1 resides within RNA-induced silencing complexes that are involved in miRNA processing. Taken together, these data indicate that IncRNA-NEAT1 regulates UCK2 by sponging miR-199a-3p as a ceRNA.

\section{LncRNA-NEAT1 sustains the growth of HCC cells under hypoxic conditions via the regulation of miR-199a-3p/UCK2}


To further evaluate the role of the Inc-NEAT1/miR-199a-3p/UCK2 axis in Inc-NEAT-1-mediated growth promotion, we repeated the MTT assays in cells transfect with UCK2 overexpression vector or miR-199a$3 p$ mimic. Under normoxic conditions, UCK2 overexpression significantly enhanced cell proliferation, while miR-199a-3p overexpression produced opposite results. Furthermore, under hypoxic condition, UCK2 knock-down or miR-199a-3p overexpression significantly neutralized the sustaining effect of IncRNA-NEAT1 on cell proliferation (Fig. 6a). The converse trend was observed in our evaluation of cell apoptosis, for which miR-199a-3p-mimic caused an increase in apoptosis under normoxic conditions, and si-UCK2 and miR-199a-3p neutralized the reduction in apoptosis levels mediated by IncRNA-NEAT1 overexpression under hypoxic conditions (Fig. 6b). A similar trend was also observed for cell cycle arrest (Fig. 6c), and these results were confirmed in HUH7 cells (Additional file 1: Supplementary Fig. 4), which suggests that UCK2 and miR-199a-3p have critical roles in IncRNA-NEAT1-induced HCC cell promotion under conditions of hypoxia.

\section{LncRNA-NEAT1 promotes HCC tumor growth through miR- 199a-3p/UCK2 in vivo}

To determine whether Inc-NEAT contributes to HCC by a similar mechanism in vivo, we evaluated the effect of IncRNA-NEAT1, sh-UCK2 and miR-199a-3p expression in xenografted mouse tumors from SNU182 cells. LncRNA-NEAT1 promoted the development of xenografted tumors (Fig. 7a), which was evidenced by larger tumor volumes (Fig. 7b) and higher tumor weights (Fig. 7c). However, co-transfection of either miR-199a-3p mimic or sh-UCK2 inhibited these promotive effects of IncRNA-NEAT1. Thus, these findings support the role of the Inc-NEAT1/miR-199a-3p/UCK2 axis in HCC tumor growth in vivo.

\section{Discussion}

Hypoxia-regulated IncRNAs play pivotal roles in the development of various cancers, including HCC, gastric cancer, and pancreatic cancer, by regulating cellular proliferation, invasion, metastasis, metabolism, and autophagy[14]. As an example, Zhao et al. found that IncRNA-MALAT1 is significantly overexpressed in HCC cells under hypoxic conditions, whereas knock-down of MALAT1 weakened the promotive effect of hypoxia on cellular proliferation, migration, and invasion[10]. Zhang et al. reported that IncRNA-PCGEM1 is induced in GC cells under hypoxic conditions and acts as an oncogenic factor[15], while up-regulation of IncRNA-BX111 in response to hypoxia promotes metastasis and progression of pancreatic cancer[9]. In the present study, we confirmed the response of IncRNA-NEAT1 to hypoxia and demonstrated that IncRNA-NEAT1 is transcriptionally regulated by HIF-1a in HCC cells. Integrated analysis of public HCC-related datasets was performed to select a group of HCC-associated miRNA-mRNA pairs that could potentially be modulated by IncRNA-NEAT1 in a ceRNA-related manner. Moreover, the regulatory effects of IncRNA-NEAT1 on the miR-199a-3p/UCK2 axis in HCC were validated both in vitro and in vivo. 
LnCRNA-NEAT1 has been established as a target for the diagnosis and treatment of various solid tumors. Elevated expression of IncRNA-NEAT1 drives tumor initiation and progression by regulating cellular growth, migration, invasiveness, epithelial-to-mesenchymal transition, and stemness. Furthermore, IncRNA-NEAT1 has been reported to affect the sensitivity to sorafenib and radiotherapy, as well as immune escape in HCC in vitro. Consistent with this role, aberrant expression of IncRNA-NEAT1 has been demonstrated in HCC and is associated with poor survival of HCC patients [11, 16-18]. Liu et al. demonstrated that high expression of IncRNA-NEAT1 in a Chinese population is an independent risk factor for poor survival of patients with HCC[19]. Furthermore, Ling et al. evaluated the expression level and clinical relevance of IncRNA-NEAT1 in HCC based on data from TCGA-LIHC and other HCC datasets from the Oncomine database, and found that IncRNA-NEAT1 is consistently up-regulated in HCC tumor tissues, though in the TCGA-LIHC dataset, IncRNA-NEAT1 was not significantly associated with overall patient survival but was significantly correlated to distant metastasis. Combined with data from in vitro experiments showing that knock-down of IncRNA-NEAT1 inhibits proliferation and induces apoptosis, Ling et al. suggested that IncRNA-NEAT1 promotes deterioration in HCC[20]. In the present study, we focused more on the up- and downstream regulatory mechanisms of IncRNA-NEAT1 in HCC rather, than on its tumor-promoting role under conventional conditions, and our results, therefore, may be increase the mechanistic understanding of IncRNA-NEAT1 in each of these prior investigations.

In general, the abnormal expression of IncRNA-NEAT1 in cancer cells is known to be caused by genetic alterations, transcription factors, DNA methylation, miRNAs, and RNA-binding proteins[21]. Emerging evidence indicates that hypoxia can modulate the expression of IncRNAs, including IncRNA-NEAT1. Upregulation of IncRNA-NEAT1 has been found in breast cancer cells under hypoxic conditions[13] and in cardiomyocytes[22], further indicating that IncRNA-NEAT1 may be a hypoxia-responsive IncRNA. However, the response of IncRNA-NEAT1 to hypoxia in HCC has not been well elucidated. Therefore, we investigated changes in IncRNA-NEAT1 expression levels in HCC cells under hypoxic conditions $\left(1 \% \mathrm{O}_{2}\right)$ or treatment with the hypoxia mimetic $\mathrm{CoCl}_{2}$. Our data verify that IncRNA-NEAT1 is induced by hypoxia. HIF-1a has been established as a predominant transcriptional regulator in response to hypoxia with the ability to binding to HREs and enhance expression of target genes, including IncRNAs[23]. By accessing the JASPAR database, we identified a potential putative HIF-1a-related HRE (5'-ACGTGC-3') located in the promoter of IncRNA-NEAT1. Knock-down of HIF-1 a eliminated the response of IncRNA-NEAT1 to hypoxia. Furthermore, the results of the ChIP and luciferase reporter assays supported the binding of HIF-1a to the promoter of IncRNA-NEAT1, suggesting that IncRNA-NEAT1 is transcriptionally induced by HIF-1a. These results provide evidence for the role of HIF-1a.and the HRE in the IncRNA-NEAT1 promoter as a mechanism that regulates IncRNA-NEAT1 expression under conditions of hypoxia.

We further demonstrated that overexpression of IncRNA-NEAT1 does not promote proliferation of HCC cells under normoxic conditions, possibly because the overactive proliferative properties of tumor cells under normal conditions may obscure the effect of IncRNA-NEAT1 overexpression by the "ceiling effect". On the other hand, the overall proliferative rate is lower under conditions of hypoxia, and though IncRNANEAT1 expression is higher, exogenous overexpression in hypoxia conditions increased the proliferation 
rate. As expected, knock-down of IncRNA-NEAT1 inhibited the growth of HCC cells, which was evident both under normoxic and hypoxic conditions. Moreover, acute hypoxia inhibited growth and promoted apoptosis and cell cycle arrest of HCC cells, and HCC cells overexpressing IncRNA-NEAT1 grew relatively faster with less apoptosis and G1 phase arrest. Therefore, these results suggest that increased IncRNANEAT1 levels sustain the growth of HCC cells under hypoxic conditions.

LncRNAs regulate gene expression in cancers through distinct mechanisms. For instance, IncRNAs may regulate target genes by specific recruitment of transcriptional activators or suppressors; by acting as decoys that bind to and block transcription factors from target genes; or by recruiting chromatinremodeling complexes as scaffolding proteins, thereby affecting target genes [24]. In tumor biology, IncRNAs primarily serve as ceRNAs that sponge tumor-promotive or tumor-suppressive miRNAs. Sponged miRNAs lose their regulatory effect on target mRNAs, which ultimately influences tumor progression[25]. Thus, we employed microarray analysis and a series of advanced online bioinformatics tools to identify potential miRNA-mRNA pairs that may interact with IncRNA-NEAT1 according to a ceRNA mechanism. The candidate miRNA-mRNA pairs were filtered according to their reported suppressive functions and predicted interactions with tumor-related genes, as well as by their down-regulated expression patterns in HCC. To obtain more evidence for the roles of candidate miRNAs in HCC, we performed pathway annotation analysis of predicted miRNA targets based on the KEGG database. The candidate miRNAs were determined to be involved in multiple tumor-related pathways. To filter HCC-related mRNA targets of IncRNA-NEAT1-miRNAs, 7 independent datasets from HCC patients with different backgrounds were used for integrated analysis using the RRA method, which strengthened the evidence. Eight candidate mRNAs were up-regulated in $\mathrm{HCC}$ tissues in the 7 datasets. To better explore the potential biological functions of the candidate mRNAs, GO enrichment analysis was performed. Enriched GO-terms, such as 'regulation of cell growth' and 'positive regulation of cell cycle', provided a potential explain of how IncRNA-NEAT1 may sustain growth of HCC cells by regulating the candidate mRNAs identified in our study. Though we could not perform a detailed analysis of all candidate miRNA-mRNA pairs in the present study, we selected UCK2, a hub target gene and impressive prognosis risk factor of HCC, for confirmation. The miRNAs that potentially regulate UCK2, including miR-199a-3p, miR-483-3p, miR-486-5p, miR-582-5p, and miR-129-5p, were considered. However, definite interactions were verified only for the IncRNA-NEAT1-miR-199a-3pUCK2 axis. The results of ChIP and luciferase analyses confirmed the binding of IncRNA-NEAT1/miR199a-3p and miR-199a-3p/UCK2, thus providing a downstream mechanism that may regulate IncRNANEAT1 function.

Previous studies have reported that miR-199a-3p acts as a tumor suppressor via various mechanisms in HCC. For instance, miR-199a-3p inhibits tumor growth in an animal model of HCC by modulating the mTOR pathway[26]. Giovannini et al. suggested that miR-199a-3p down-regulation is a common characteristic of HCC and that miR-199a-3p regulates E-cadherin expression through Notch1[27], and Guan et al. reported that miR-199a-3p represses tumorigenesis in HCC by targeting the ZHX1/PUMA pathway[28]. In the present study, the suppressive role of miR-199a-3p was confirmed and expression changes of IncRNA-NEAT1 were shown to induce alteration of miR-199a-3p in HCC cells. Furthermore, 
luciferase reporter and RIP assay demonstrated that IncRNA-NEAT1 sponges miR-199a-3p, which is consistent with our other bioinformatics and experimental data.

Functionally, UCK2 is a pyrimidine ribonucleotide kinase that catalyzes phosphorylation of uridine to uridine monophosphate and cytidine to cytidine monophosphate. Overexpression of UCK2 is regarded as an indicator of unfavorable prognosis in various cancers, including HCC, pancreatic cancer, breast cancer, and lung cancer[29-32]. However, few studies have revealed the detailed mechanisms underlying the regulation of UCK2. Zhou et al. found that UCK2 promotes metastasis via up-regulation of MMP2/9 expression and activation of STAT3 signaling[33]. The upstream mechanisms of UCK2, especially those involved with IncRNA/miRNA, had not been clarified prior to this study. Therefore, we confirmed the growth-promotive effect of UCK2 in HCC cells and demonstrated that UCK2 is regulated by IncRNANEAT1/miR-199a-3p. Most importantly, IncRNA-NEAT1 was shown to function under hypoxic conditions partly through miR-199a-3p/UCK2. Moreover, an animal model was used to further explore the role and regulatory relationship of IncRNA-NEAT1/miR-199a-3p/UCK2. As a limitation of this study, some miRNAs and mRNAs that may be also controlled by IncRNA-NEAT1 were not validated so that we could focus our efforts on validating the IncRNA-NEAT1/miR-199a-3p/UCK2 axis. These miRNAs or mRNAs should be investigated in future studies.

\section{Conclusions}

In conclusion, we identified IncRNA-NEAT1 as a hypoxia-responsive IncRNA in HCC that sustains the growth of HCC cells under hypoxic conditions. LncRNA-NEAT1 may regulate a panel of HCC-associated mRNAs by interacting with tumor-suppressive miRNAs in HCC. The roles of IncRNA-NEAT1/miR-199a3p/UCK2 were validated, which indicated that IncRNA-NEAT1 and its downstream miRNAs/mRNAs may contribute to the progression of $\mathrm{HCC}$ in hypoxic microenvironments and, therefore, are potential targets for novel therapeutic strategies for HCC.

\section{Abbreviations}




\begin{tabular}{ll} 
CDNA & complementary DNA \\
\hline ChIP & chromatin immunoprecipitation \\
\hline HCC & hepatocellular carcinoma \\
\hline HIF & hypoxia inducible factor \\
\hline HRE & hypoxia response element \\
\hline LncRNA & long noncoding RNAs \\
\hline miRNA & microRNA \\
\hline NEAT1 & nuclear paraspeckle assembly transcript 1 \\
\hline RIP & RNA immunoprecipitation \\
\hline TCGA & The Cancer Genome Atlas
\end{tabular}

\section{Declarations}

\section{Ethics approval and consent to participate}

The animal experiments were performed in strict accordance with the guidelines of the Research Animal Care Committee Zhengzhou University.

\section{Consent for publication}

Not applicable.

\section{Competing interests}

The authors declare that they have no competing interests.

\section{Funding}

This study was supported by the Nanchong Municipality-University Strategic Cooperative Science and Technology Project (project No.:18SXHZ0255) and the International Cooperation in Science and Technology/ Research and Development Program of Sichuan Province (project No.:20GJHZ0231)

\section{Author contributions}

Study design: ZQN and LJ; acquisition of data: CQ, XMT, HXY and HXT; analysis and interpretation of data: ZQN, and CQ; drafting of the manuscript: ZQN, CQ; statistical analysis: ZQN, CQ; funding and study supervision: LJ and LLP. All authors read and approved the final manuscript. 
None.

\section{References}

1. Bray F, Ferlay J, Soerjomataram I, Siegel RL, Torre LA, Jemal A: Global cancer statistics 2018: GLOBOCAN estimates of incidence and mortality worldwide for 36 cancers in 185 countries. CA: a cancer journal for clinicians2018; 68: 394-424.

2. Kulik L, El-Serag HB: Epidemiology and Management of Hepatocellular Carcinoma. Gastroenterology2019; 156: 477-491.e471.

3. Llovet JM, Montal R, Sia D, Finn RS: Molecular therapies and precision medicine for hepatocellular carcinoma. Nature reviews. Clinical oncology2018; 15: 599-616.

4. Xiong XX, Qiu XY, Hu DX, Chen XQ: Advances in Hypoxia-Mediated Mechanisms in Hepatocellular Carcinoma. Molecular pharmacology2017; 92: 246-255.

5. Lin D, Wu J: Hypoxia inducible factor in hepatocellular carcinoma: A therapeutic target. World J Gastroenterol2015; 21: 12171-12178.

6. Wong CC-L, Kai AK-L, Ng IO-L: The impact of hypoxia in hepatocellular carcinoma metastasis. Front Med2014; 8: 33-41.

7. Shih J-W, Kung H-J: Long non-coding RNA and tumor hypoxia: new players ushered toward an old arena. J Biomed Sci2017; 24: 53-53.

8. Choudhry H, Harris AL: Advances in Hypoxia-Inducible Factor Biology. Cell Metab2018; 27: 281-298.

9. Deng S-J, Chen H-Y, Ye Z, et al.: Hypoxia-induced LncRNA-BX111 promotes metastasis and progression of pancreatic cancer through regulating ZEB1 transcription. Oncogene2018; 37: 58115828.

10. Zhao ZB, Chen F, Bai XF: Long Noncoding RNA MALAT1 Regulates Hepatocellular Carcinoma Growth Under Hypoxia via Sponging MicroRNA-200a. Yonsei Med J2019; 60: 727-734.

11. Zhu L, Yang N, Li C, Liu G, Pan W, Li X: Long noncoding RNA NEAT1 promotes cell proliferation, migration, and invasion in hepatocellular carcinoma through interacting with miR-384. J Cell Biochem2018; 120: 1997-2006.

12. Tu J, Zhao Z, Xu M, Lu X, Chang L, Ji J: NEAT1 upregulates TGF- $\beta 1$ to induce hepatocellular carcinoma progression by sponging hsa-mir-139-5p. J Cell Physiol2018; 233: 8578-8587.

13. Choudhry $\mathrm{H}$, Albukhari $\mathrm{A}$, Morotti $\mathrm{M}$, et al.: Tumor hypoxia induces nuclear paraspeckle formation through HIF-2a dependent transcriptional activation of NEAT1 leading to cancer cell survival. Oncogene2015; 34: 4482-4490.

14. Huang L, Wang W, Hu Z, Guan C, Li W, Jiang X: Hypoxia and IncRNAs in gastrointestinal cancers. Pathol Res Pract2019; 215: 152687-152687.

15. Zhang J, Jin HY, Wu Y, et al.: Hypoxia-induced LncRNA PCGEM1 promotes invasion and metastasis of gastric cancer through regulating SNAI1. Clin TransI Oncol2019; 21: 1142-1151. 
16. Chen S, Xia X: Long noncoding RNA NEAT1 suppresses sorafenib sensitivity of hepatocellular carcinoma cells via regulating miR-335-c-Met. J Cell Physiol2019: 10.1002/jcp.27567.

17. Yan K, Fu Y, Zhu N, et al.: Repression of IncRNA NEAT1 enhances the antitumor activity of CD8(+)T cells against hepatocellular carcinoma via regulating miR-155/Tim-3. Int J Biochem Cell Biol2019; 110: 1-8.

18. Chen X, Zhang N: Downregulation of IncRNA NEAT1_2 radiosensitizes hepatocellular carcinoma cells through regulation of miR-101-3p/WEE1 axis. Cell Biol Int2019; 43: 44-55.

19. Liu Z, Chang Q, Yang F, et al.: Long non-coding RNA NEAT1 overexpression is associated with unfavorable prognosis in patients with hepatocellular carcinoma after hepatectomy: A Chinese population-based study. Eur J Surg Oncol2017; 43: 1697-1703.

20. Ling Z-A, Xiong D-D, Meng R-M, et al.: LncRNA NEAT1 Promotes Deterioration of Hepatocellular Carcinoma Based on In Vitro Experiments, Data Mining, and RT-qPCR Analysis. Cell Physiol Biochem2018; 48: 540-555.

21. Peng WX, Koirala P, Mo YY: LncRNA-mediated regulation of cell signaling in cancer. Oncogene2017; 36: 5661-5667.

22. Wu H-J, Tang G-M, Shao P-Y, et al.: Long non-coding RNA NEAT1 modulates hypoxia/reoxygenationinduced cardiomyocyte injury via targeting microRNA-520a. Exp Ther Med2019; 18: 2199-2206.

23. Gómez-Maldonado L, Tiana M, Roche 0 , et al.: EFNA3 long noncoding RNAs induced by hypoxia promote metastatic dissemination. Oncogene2015; 34: 2609-2620.

24. Vishnubalaji R, Shaath H, Elango R, Alajez NM: Noncoding RNAs as potential mediators of resistance to cancer immunotherapy. Semin Cancer Biol2019: S1044-1579X(1019)30223-30228.

25. Wei L, Wang X, Lv L, et al.: The emerging role of microRNAs and long noncoding RNAs in drug resistance of hepatocellular carcinoma. Mol Cancer2019; 18: 147-147.

26. Callegari E, D'Abundo L, Guerriero P, et al.: miR-199a-3p Modulates MTOR and PAK4 Pathways and Inhibits Tumor Growth in a Hepatocellular Carcinoma Transgenic Mouse Model. Mol Ther Nucleic Acids2018; 11: 485-493.

27. Giovannini C, Fornari F, Dallo R, et al.: MiR-199-3p replacement affects E-cadherin expression through Notch1 targeting in hepatocellular carcinoma. Acta Histochem2018; 120: 95-102.

28. Guan J, Liu Z, Xiao M, et al.: MicroRNA-199a-3p inhibits tumorigenesis of hepatocellular carcinoma cells by targeting ZHX1/PUMA signal. Am J Transl Res2017; 9: 2457-2465.

29. Huang S, Li J, Tam NL, et al.: Uridine-cytidine kinase 2 upregulation predicts poor prognosis of hepatocellular carcinoma and is associated with cancer aggressiveness. Mol Carcinog2019; 58: 603615.

30. El Hassouni B, Infante J, Mantini G, et al.: Uridine Cytidine Kinase 2 as a Potential Biomarker for Treatment with RX-3117 in Pancreatic Cancer. Anticancer Res2019; 39: 3609-3614.

31. Shen G, He P, Mao Y, et al.: Overexpression of Uridine-Cytidine Kinase 2 Correlates with Breast Cancer Progression and Poor Prognosis. J Breast Cancer2017; 20: 132-141. 
32. Wu Y, Jamal M, Xie T, et al.: Uridine-cytidine kinase 2 (UCK2): A potential diagnostic and prognostic biomarker for lung cancer. Cancer Sci2019; 110: 2734-2747.

33. Zhou Q, Jiang $H$, Zhang J, et al.: Uridine-cytidine kinase 2 promotes metastasis of hepatocellular carcinoma cells via the Stat3 pathway. Cancer Manag Res2018; 10: 6339-6355.

\section{Figures}

a

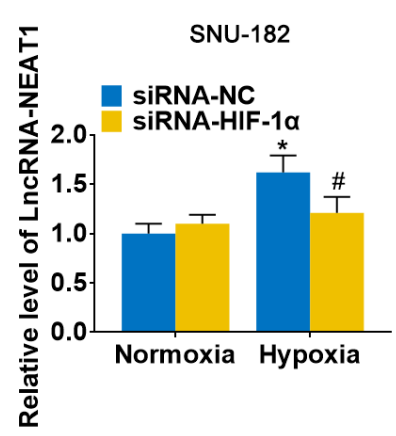

C

TCGA-LIHC

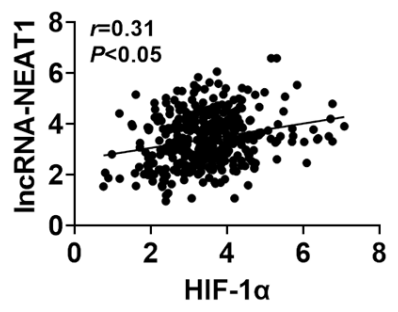

e

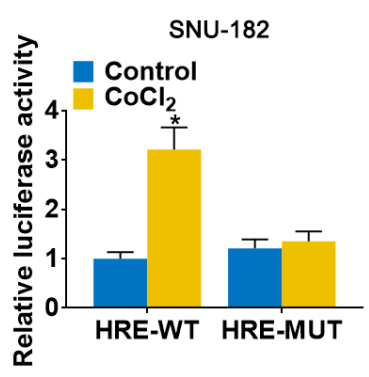

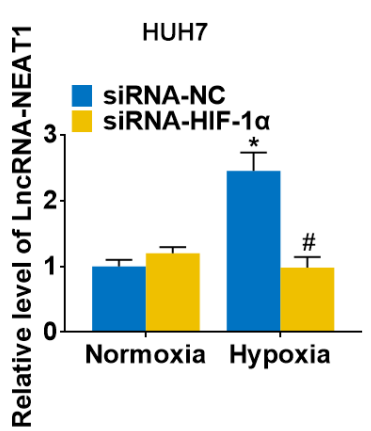

d b
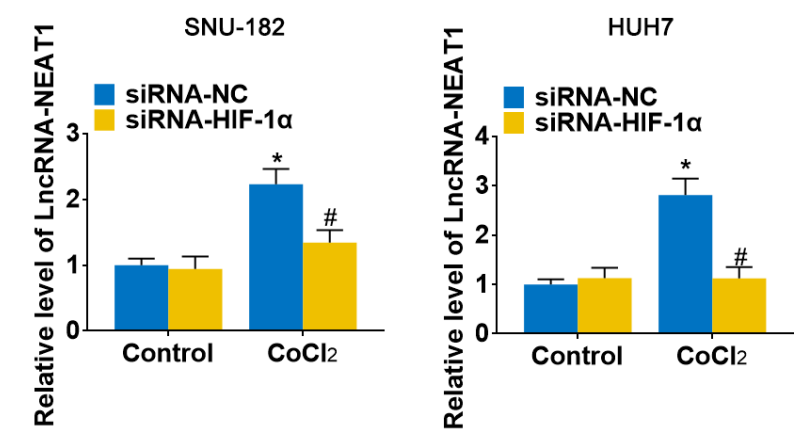

SNU-182

Input HIF-1a lgG

$-483$

5' GGACGTGCCC 3'

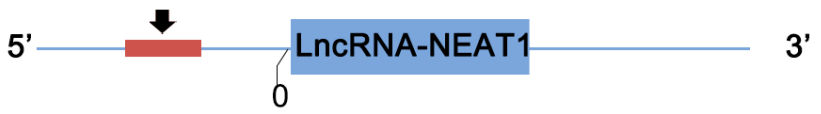

96 bp $\longrightarrow$

HUH7

Input HIF-1a IgG

96 bp
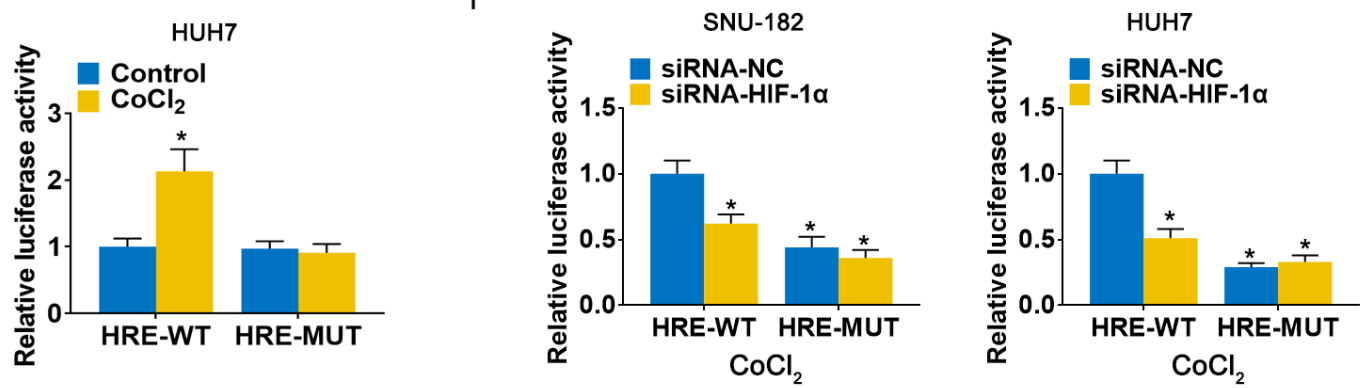

Figure 1

LncRNA-NEAT1 expression is enhanced by hypoxia via transcriptional regulation of HIF-1a in HCC cells. (a-b) HIF-1 a knock-down and control SNU-182 and HUH7 cells were cultured for $24 \mathrm{~h}$ under normoxic versus hypoxic conditions (Panel a) or without and with $\mathrm{CoCl} 2$ treatment (Panel b). Changes in the expression level of IncRNA-NEAT1 were detected by qRT-PCR. (c) The correlation of HIF-1a with Inc-NEAT1 in tumor tissues from TCGA-LIHC is shown. (d) Left: A putative HRE (ACGTGC) was identified in the promoter of IncRNA-NEAT1. Right: Binding of HIF-1a to the HRE (ACGTGC) was validated by ChIP assay in SNU-182 and HUH7 cells. HIF-1a antibody or IgG was added to the reaction. DNA fragments were amplified and analyzed by qRT-PCR with specific primers. (e) SNU-182 and HUH7 cells were transfected 
with a luciferase reporter containing the WT or mutant putative HRE (ACGTGC) sequence. Cells were treated with $\mathrm{CoCl} 2$ for $24 \mathrm{~h}$, where indicated, and relative luciferase activity was detected. (f) HIF-1a knock-down SNU-182 and HUH7 cells were transfected with a luciferase reporter containing the WT or mutant putative HRE (ACGTGC) sequence. Cells were treated with $\mathrm{CoCl} 2$ for $24 \mathrm{~h}$, and relative luciferase activity was detected. In Panels $a$ and $b,{ }^{*} \mathrm{P}<0.05$ compared with normoxia condition or control. $\# \mathrm{P}<0.05$ compared with siRNA-negative control (siRNA-NC). In Panels e and $f,{ }^{*}<0.05$ compared with normoxia control or siRNA-NC.
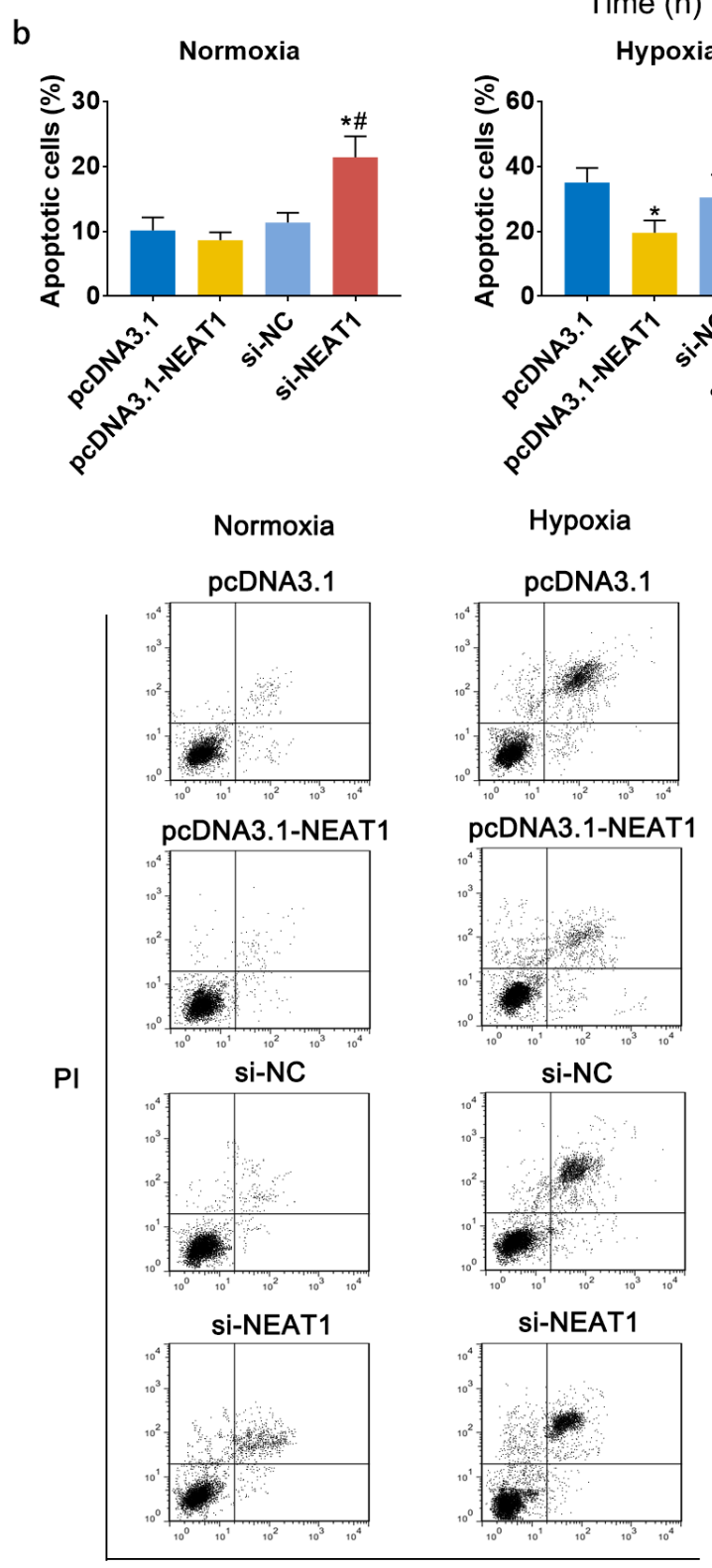

Annexin- V
Hypoxia

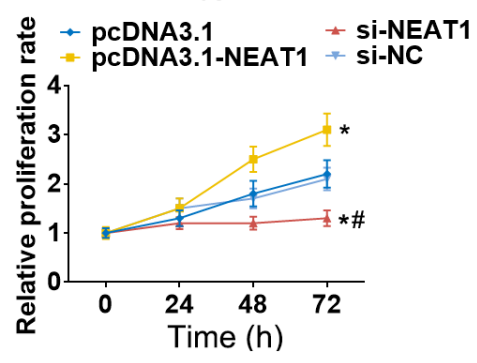

C
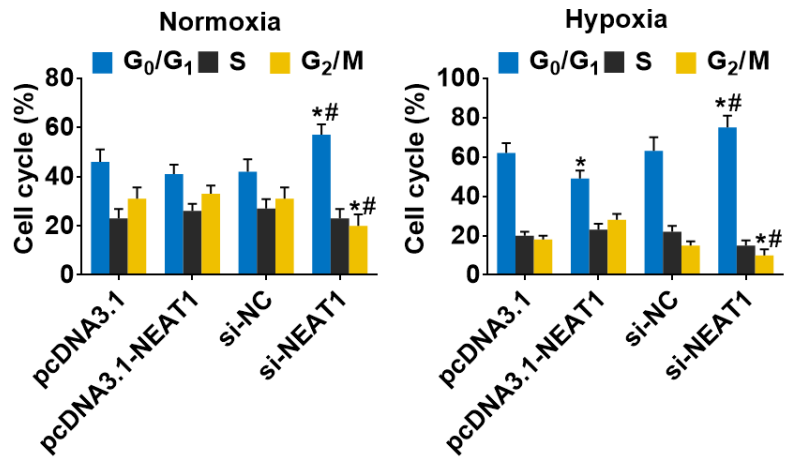

Hypoxia

pcDNA3.1

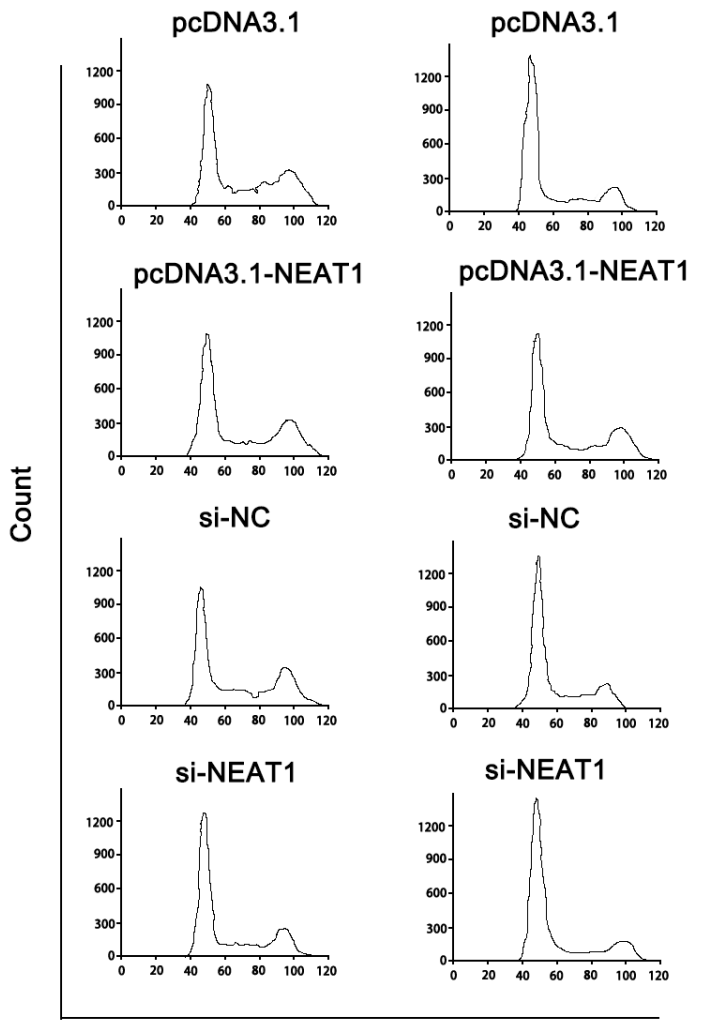

PI 


\section{Figure 3}

LncRNA-NEAT1 sustains the growth of SNU-182 cells under hypoxic conditions. (a) The expression of IncRNA-NEAT1 was modulated by transfection with the pcDNA3.1-NEAT1 vector or siRNA-NEAT1. After transfection for $24 \mathrm{~h}$, cells were cultured under hypoxic or normoxic conditions. Cell viability was detected using CCK-8 assay and used to estimate proliferation. (b-c) The roles of IncRNA-NEAT1 on apoptosis (Panel b) and the cell cycle (Panel c) in SNU-182 cells under hypoxic and normoxic conditions were assessed by flow cytometry. ${ }^{*} \mathrm{P}<0.05$ compared with transfection negative control. $\# \mathrm{P}<0.05$ compared with pcDNA3.1-NEAT1.

A

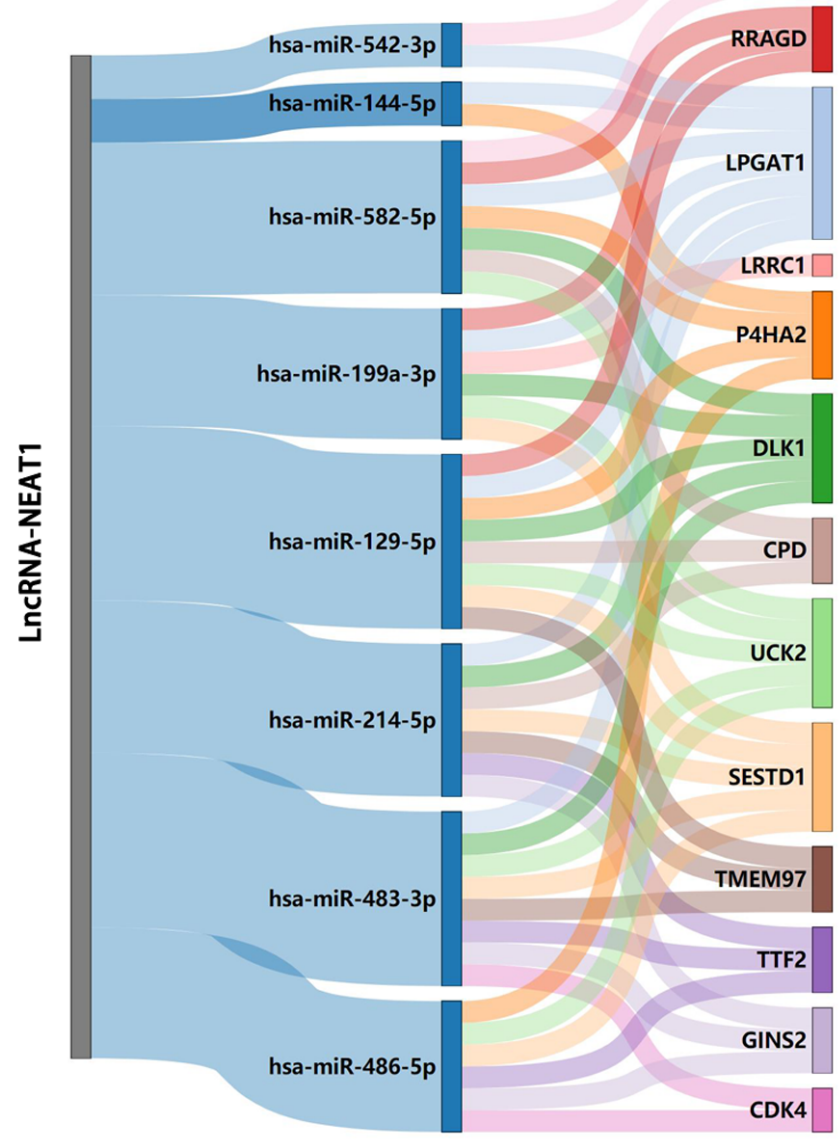

B
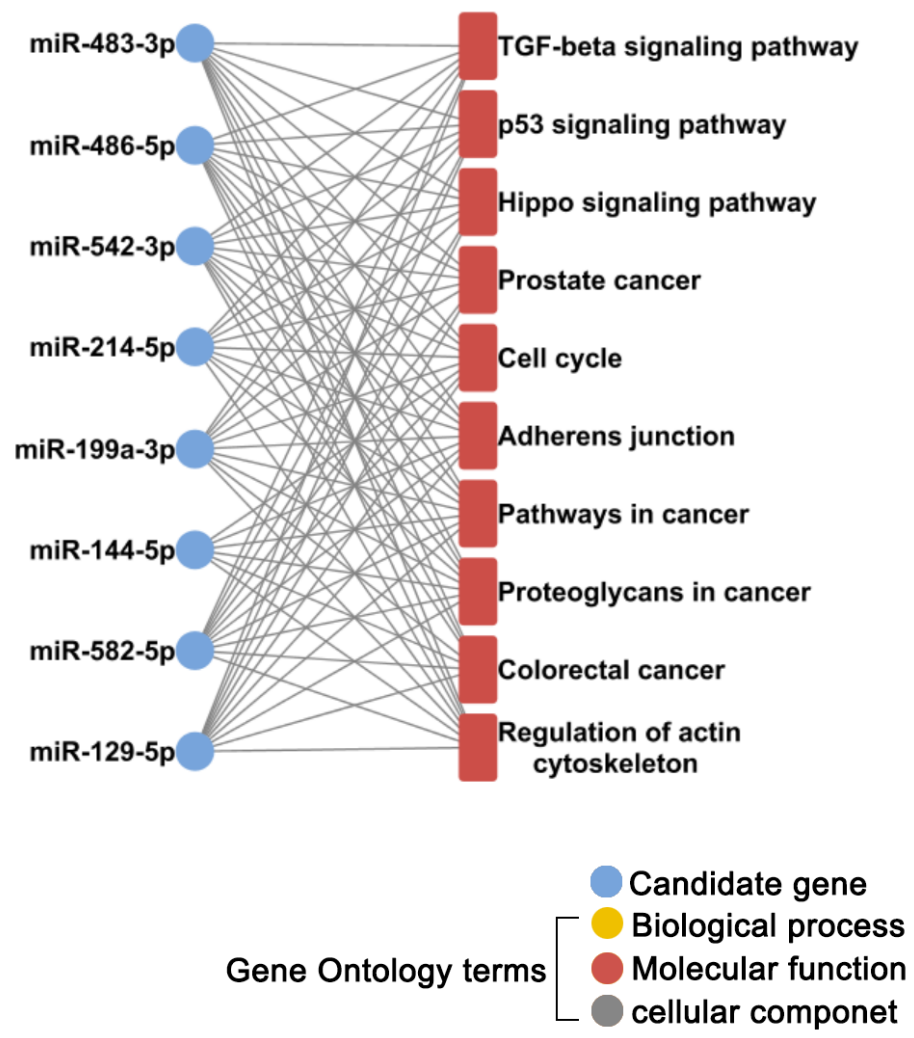

perinuclear region of cytoplasm

C

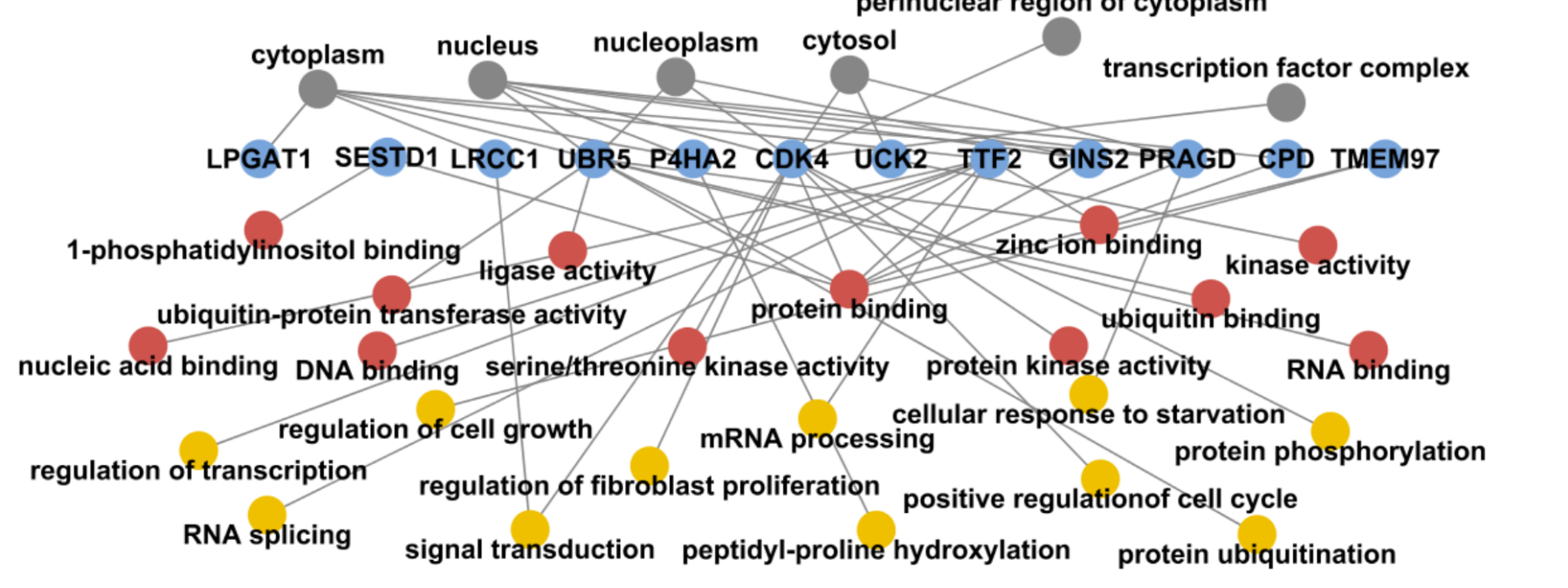




\section{Figure 5}

Microarray and integrative bioinformatics analysis of HCC-associated miRNA-mRNA pairs that are potentially regulated by IncRNA-NEAT1. (a) Differentially expressed miRNAs and mRNAs in Inc-NEAT1 knock-down SNU-182cells were identified by microarray analysis. According to the ceRNA theory, miRNAs that may be sponged by IncRNA-NEAT1 and their possible target mRNAs were also predicted by bioinformatics analysis and filtered according to their expression patterns in 7 public HCC-related datasets. Candidate miRNA-mRNA pairs that passed each of these filters are shown in the Sankey diagram. The miRNAs that are shown are predicted to act as suppressors in HCC. (bb) KEGG pathway enrichment analysis for the 8 candidate microRNA's target genes. (c) GO enrichment analysis for the 13 candidate genes. GO-biological process terms, -molecular function terms and -cellular component terms are shown. 

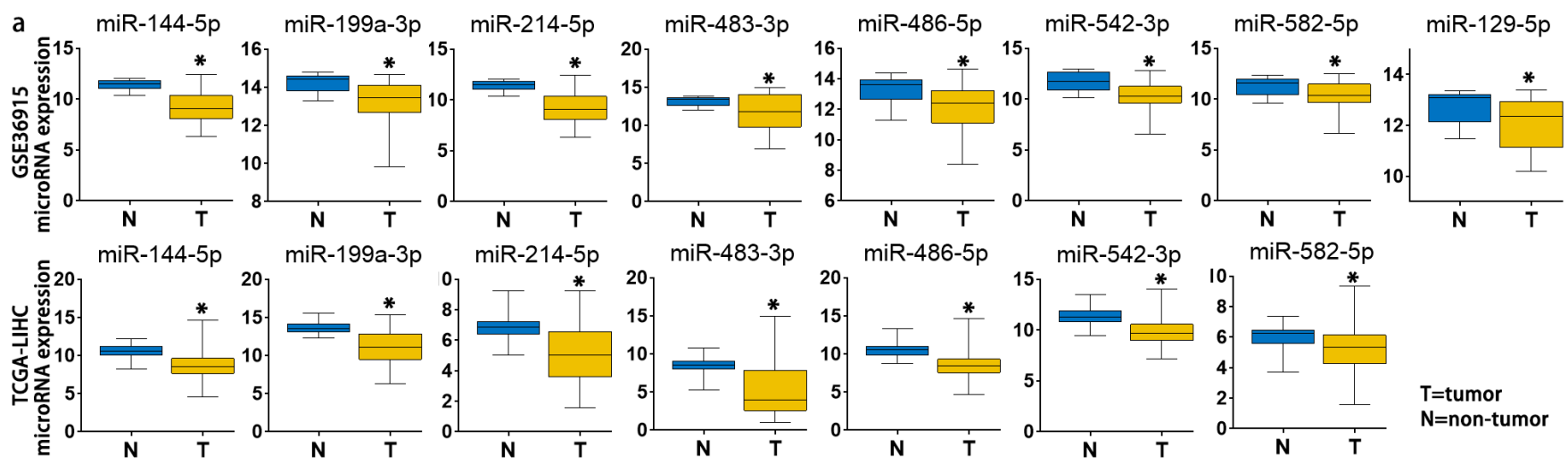

miR-582-5p

b
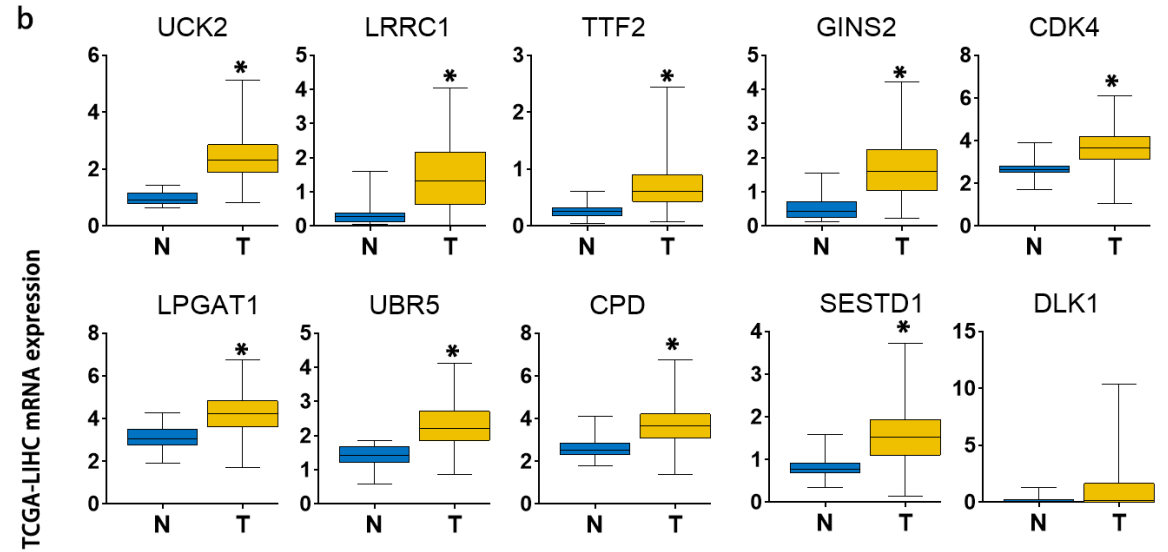

C
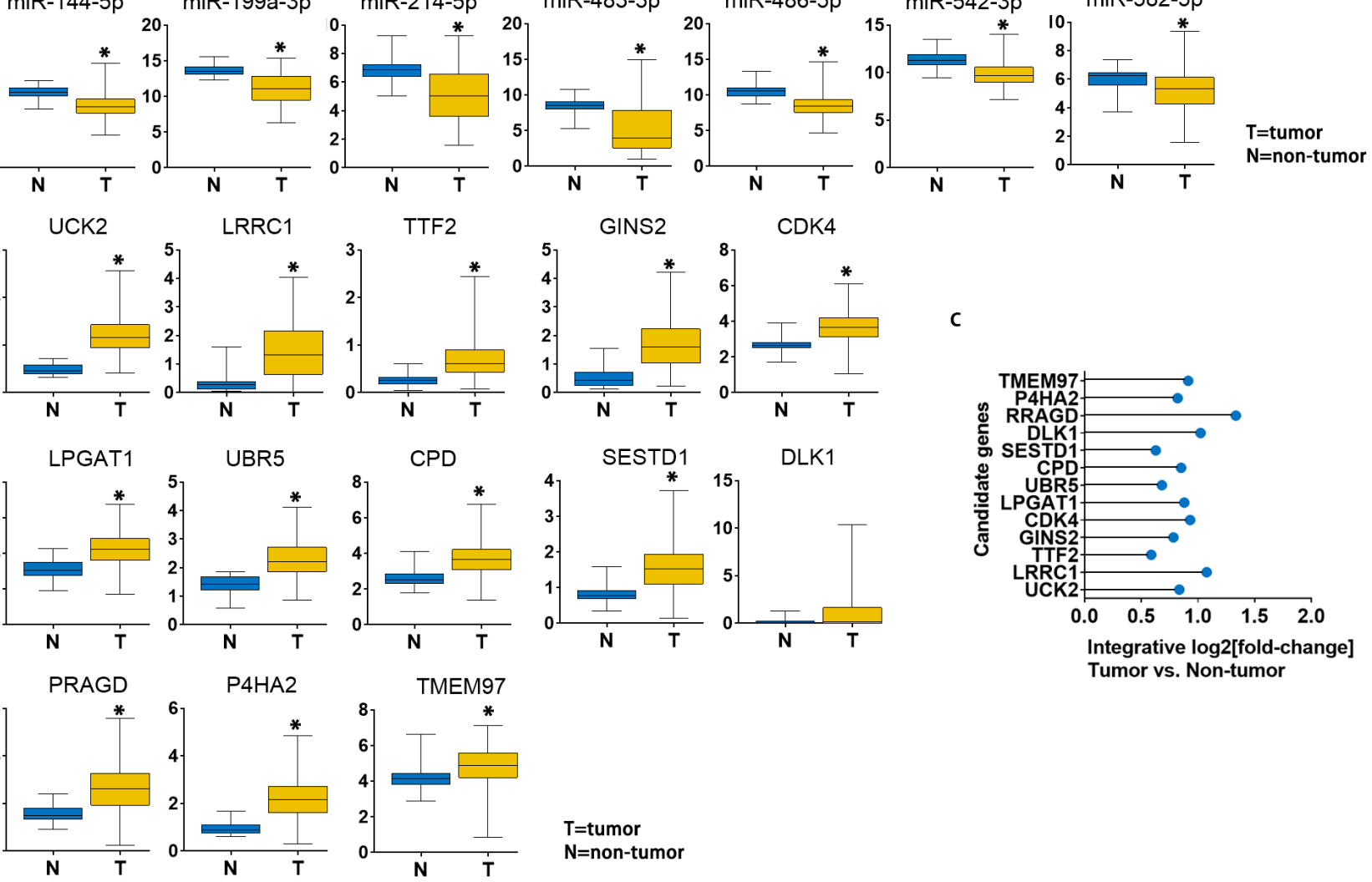

T=tumor

$\mathrm{N}=$ non-tumor

d
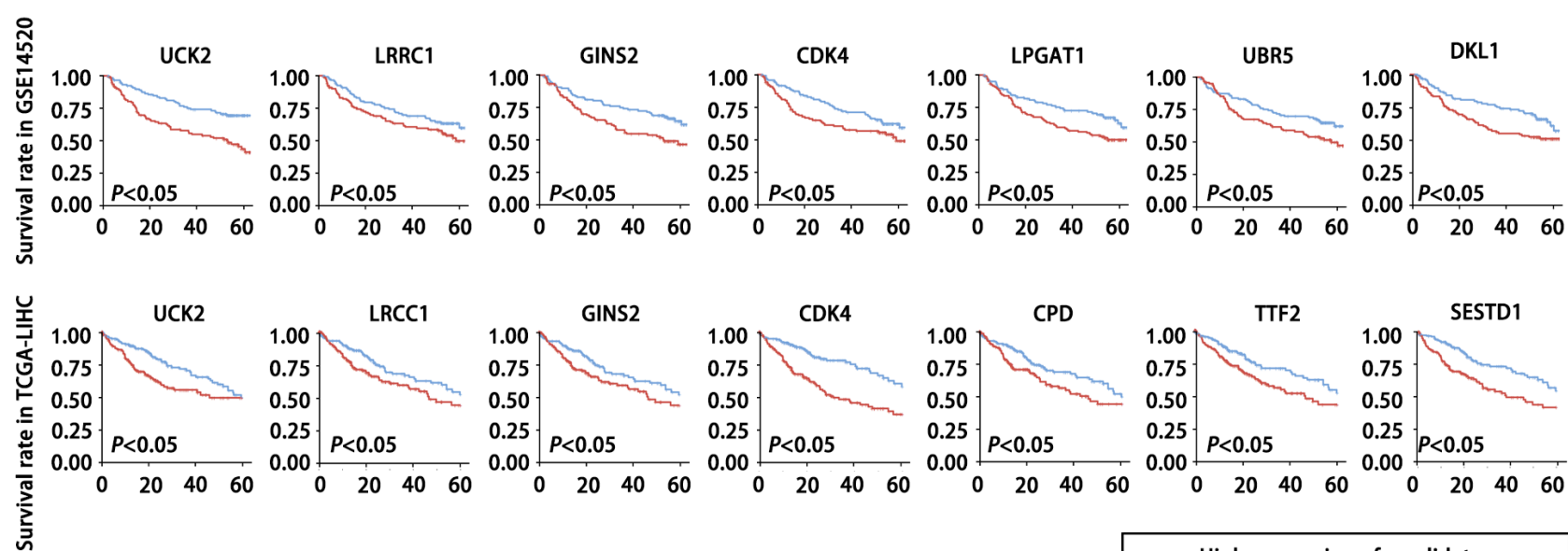

Survival in month
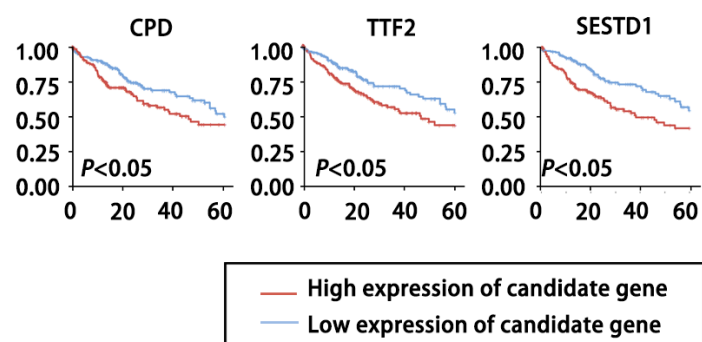

\section{Figure 7}

Expression changes and survival relevance of HCC-associated miRNAs and mRNAs that are potentially regulated by IncRNA-NEAT1. (a) Expression differences of 8 candidate miRNAs between tumor and nontumor tissues. Data are from the GSE36915 and TCGA-LIHC datasets. (b) Expression difference of 13 candidate mRNAs between tumor and non-tumor tissues in the TCGA-LIHC dataset. (c) Integrative fold change of 13 candidate mRNAs in tumor tissues as compared with non-tumor tissue in 7 independent 

Aggregation method. (d) The associations of the 13 candidate genes with survival are shown using Kaplan-Meier survival curves based on data from the GSE14520 and TCGA-LIHC datasets. ${ }^{*}<<0.05$ compared with normal tissue.

a
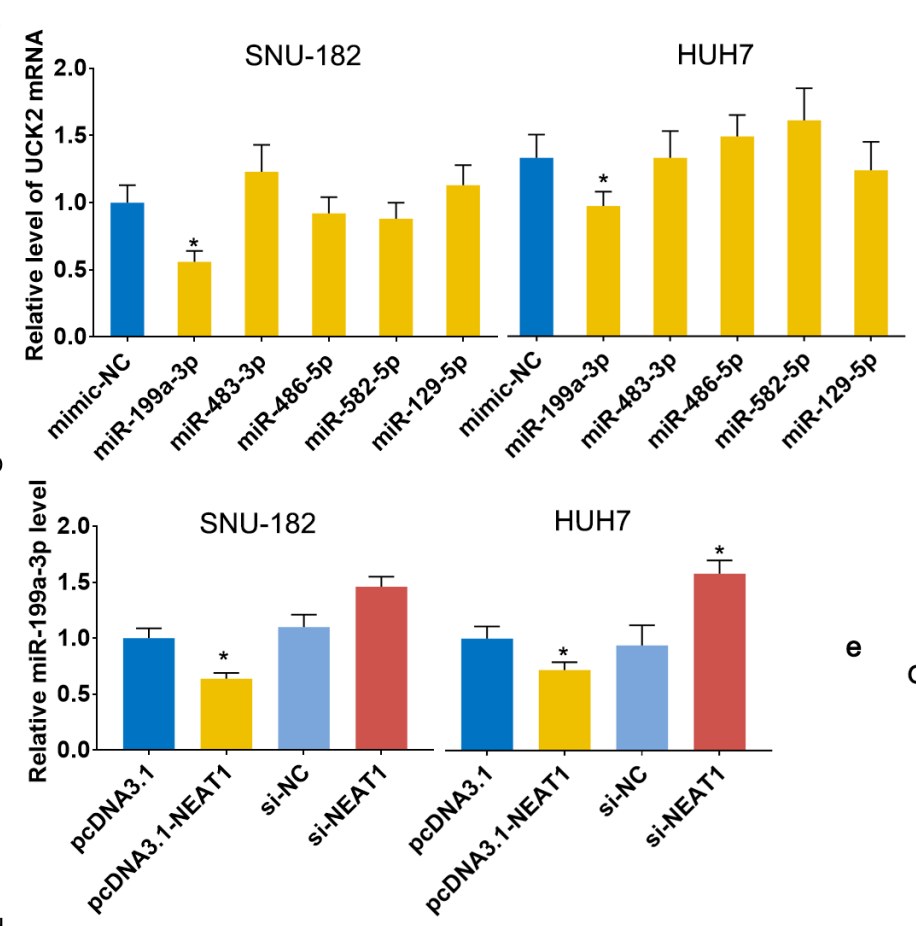

d

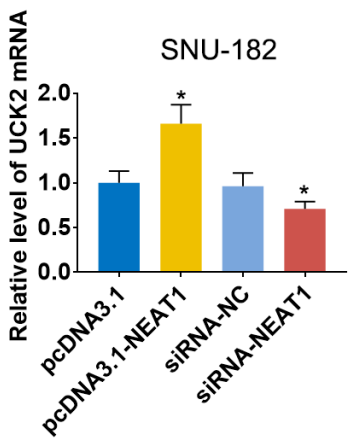

f

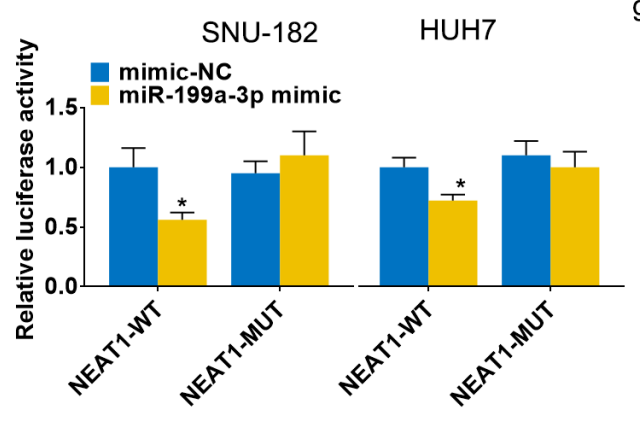

NEAT1-WT:5' cccUCCACGUGUACACUACUGa 3' miRNA-199a-3p : 3' auugGUACACGUCUGAUGACa 5'

NEAT1-MUT:5' cccUCCACGUGUACGAGGAACa 3'

e b
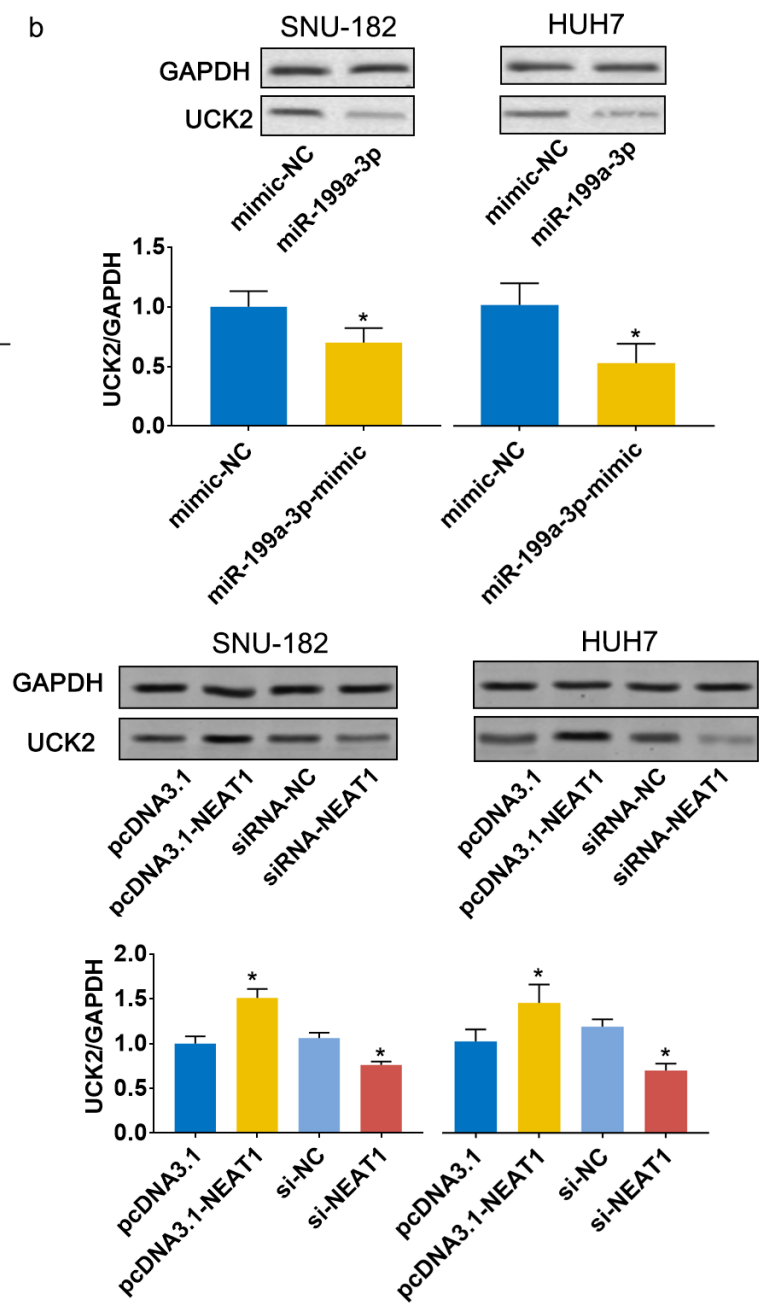

$\mathrm{h}$

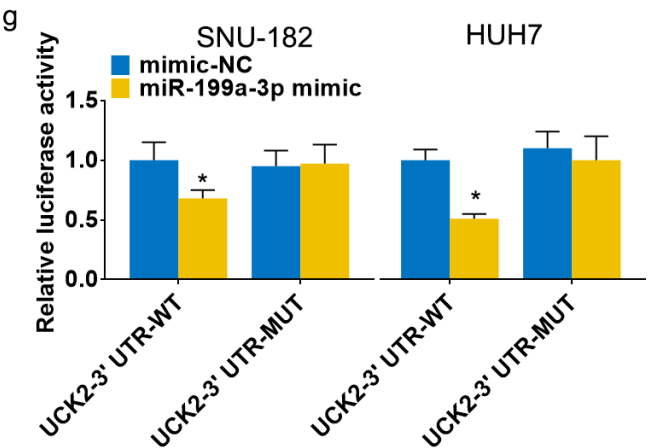

UCK2-3'UTR-WT: 5' UaACAAAACUGUGCCAACUACUACUGg 3' miRNA-199a-3p : 3' auUGGUUACACGUCUGAUGACa 5' UCK2-3'UTR-MUT: 5' uaACAAAACUGUGCCAACUCAGGAGAg 3'

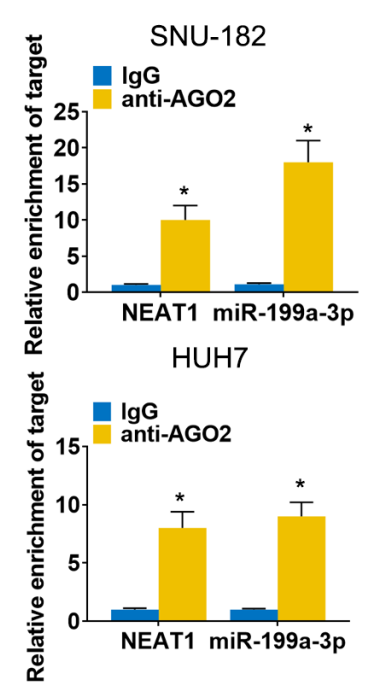

Figure 9 
LncRNA-NEAT1 regulates UCK2 by sponging miR-199a-3p in HCC cells. (a-b) SNU-182 and HUH7 cells were transfected with miR-mimics of miR-199a-3p, miR-483-3p, miR-486-5p, miR-582-5p, or miR-129-5p. After $48 \mathrm{~h}$, the mRNA and protein expression changes of UCK2 were determined by qRT-PCR (Panel a) and western blot analyses (Panel b). (c) SNU-182 and HUH7 cells were transfected with pcDNA3.1-NEAT1, siRNA-NEAT1, or their respective controls. After $48 \mathrm{~h}$ of transfection, changes in miR-199a-3p expression levels were detected by qRT-PCR. (d-e) SNU-182 and HUH7 cells were transfected with pcDNA3.1-NEAT1, siRNA-NEAT1, or their respective controls. After $48 \mathrm{~h}$ of transfection, changes in UCK2 mRNA (Panel D) and protein expression levels (Panel E) were determined by qRT-PCR and western blot analyses. (f-g) The binding of IncRNA-NEAT1 to miR-199a-3p (Panel f) and miR-199a-3p to the 3' untranslated region of UCK2 (Panel g) were verified by luciferase reporter assay. Wild-type and Mutant sequences of NEAT1 or UCK2 3' UTR are shown at the bottom. (h) RIP assay was performed to further confirm whether IncRNANEAT1 regulates miR-199a-3p as a ceRNA. Cell lysates collected from SNU-182 and HUH7 cells were incubated with antibodies against AGO2 or IgG. Enrichment of IncRNA-NEAT1 and miR-199a-3p in purified RNA was detected by qRT-PCR. In Panels a-g, * $P<0.05$ compared with transfection negative control. In Panel $h, * P<0.05$ compared with IgG control. 

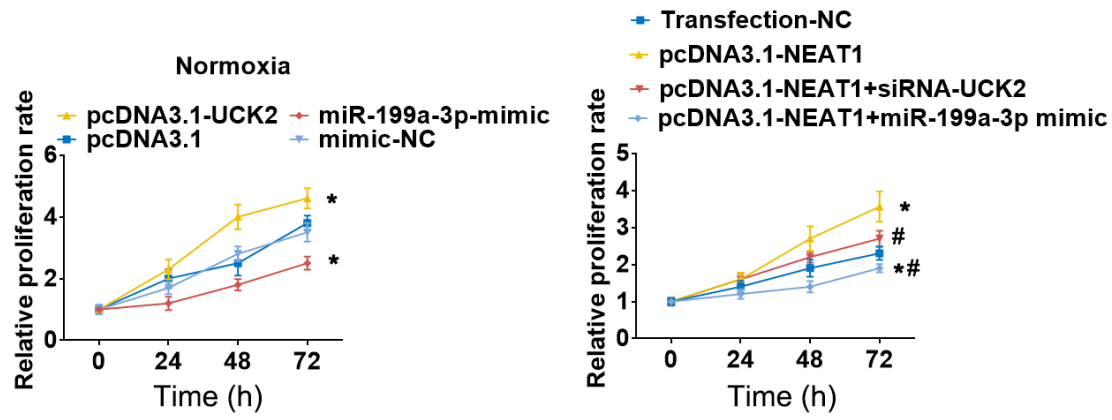

b
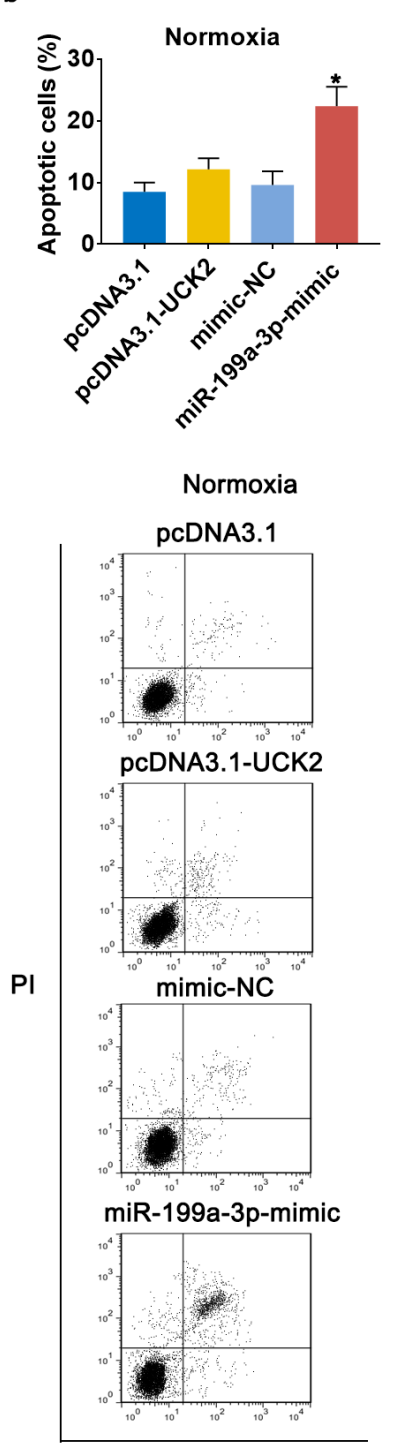

Annexin- V
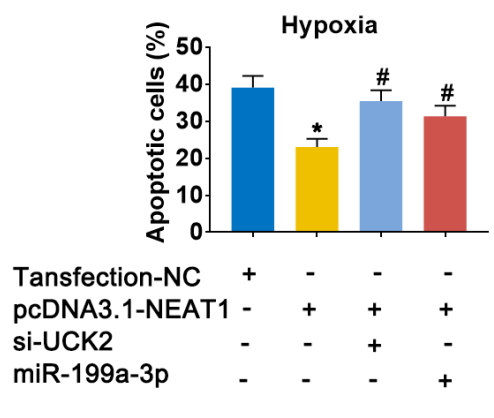

Hypoxia

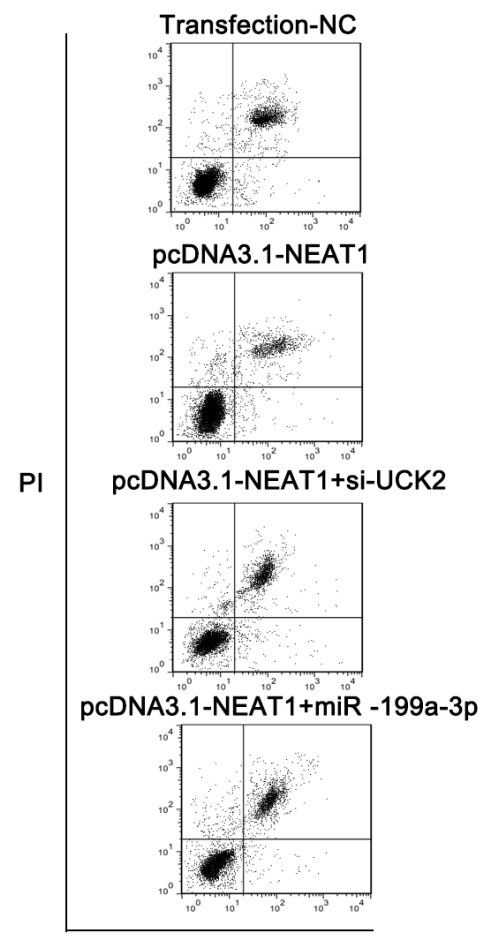

Annexin- V

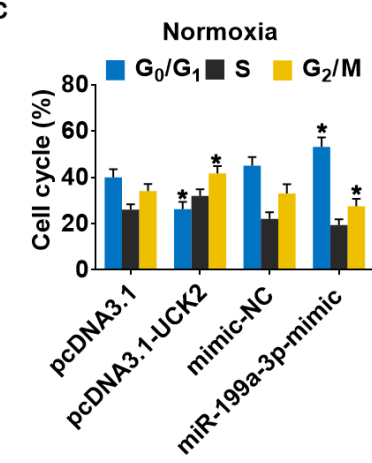

Normoxia

pcDNA3.1

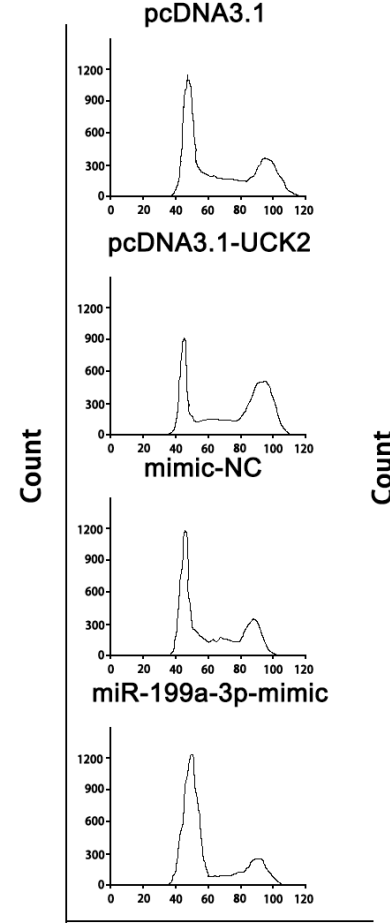

PI
Hypoxia

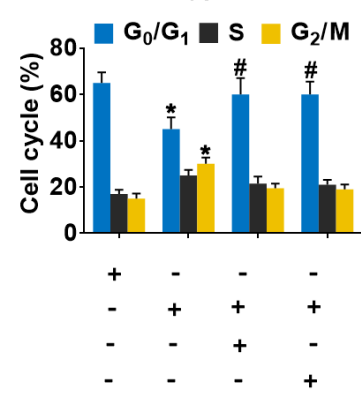

Hypoxia

Transfection-NC

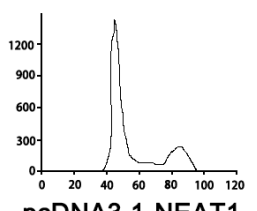

pcDNA3.1-NEAT1

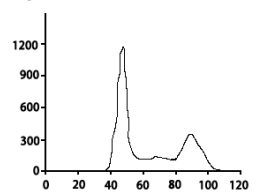

pcDNA3.1-NEAT1+si-UCK2

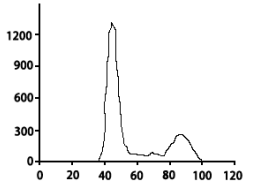

pcDNA3.1-NEAT1+miR -199a-3p

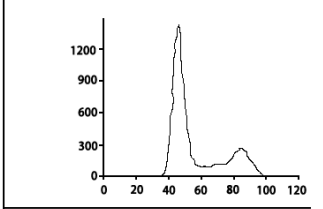

PI

Figure 11

LncRNA-NEAT1 sustains the growth of SNU-182 cells under hypoxic conditions by regulating miR-199a3p/UCK2. (a) The expression levels of miR-199a-3p and UCK2 in SNU-182 were up-regulated by transfection under normoxic conditions; or miR-199a-3p or siRNA-UCK2 were co-transfected with pcDNA3.1-NEAT1 in SNU-182 cells under hypoxic conditions. The cell proliferation changes were determined by CCK-8 assay. (b-c) The effects of miR-199a-3p and UCK2 on apoptosis (Panel b) and the 
cycle (Panel c) of SNU-182 cells were determined by flow cytometry. ${ }^{*} \mathrm{P}<0.05$ compared with transfection negative control. \#P<0.05 compared with pcDNA3.1-NEAT1.

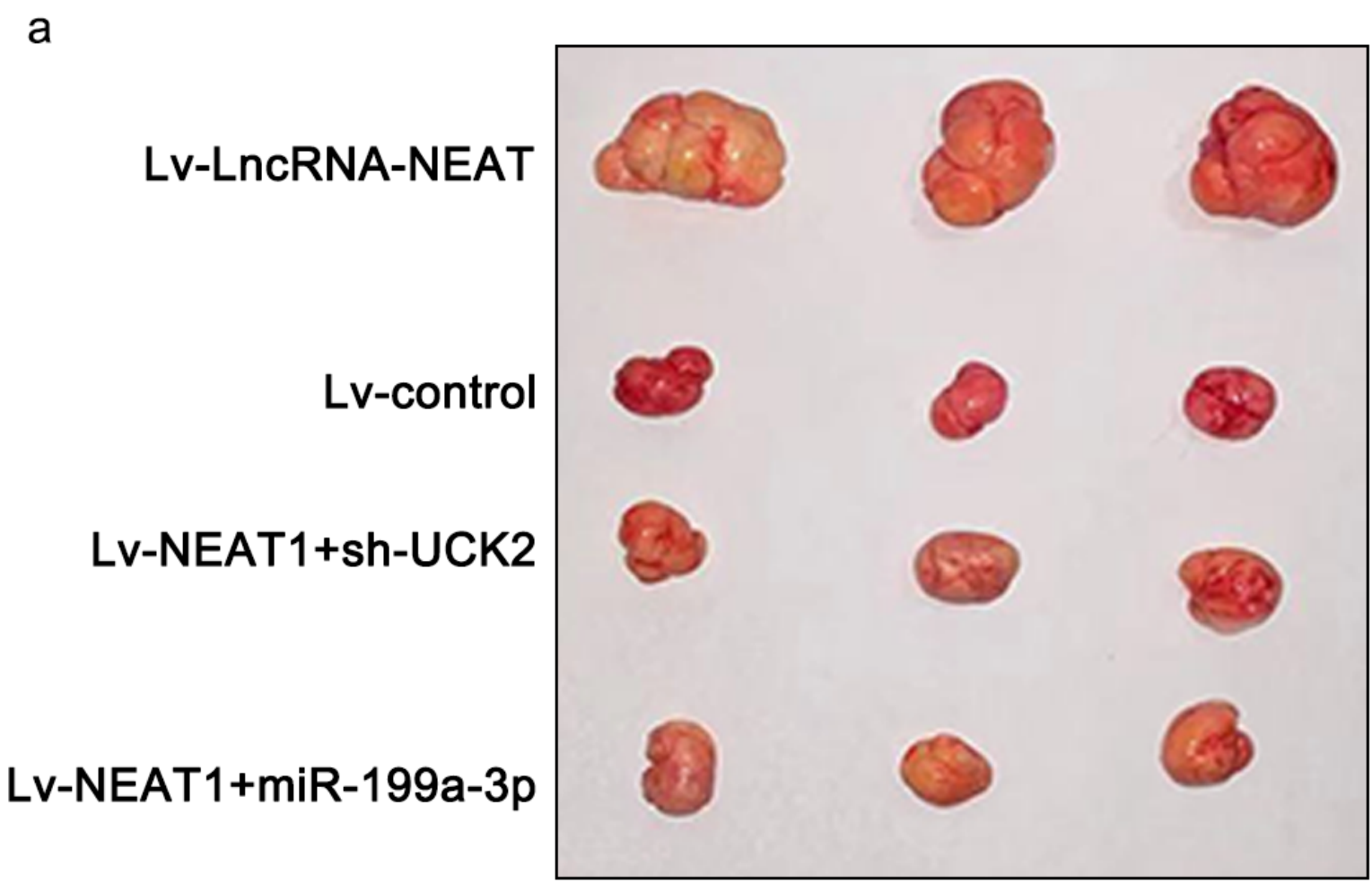

b

C
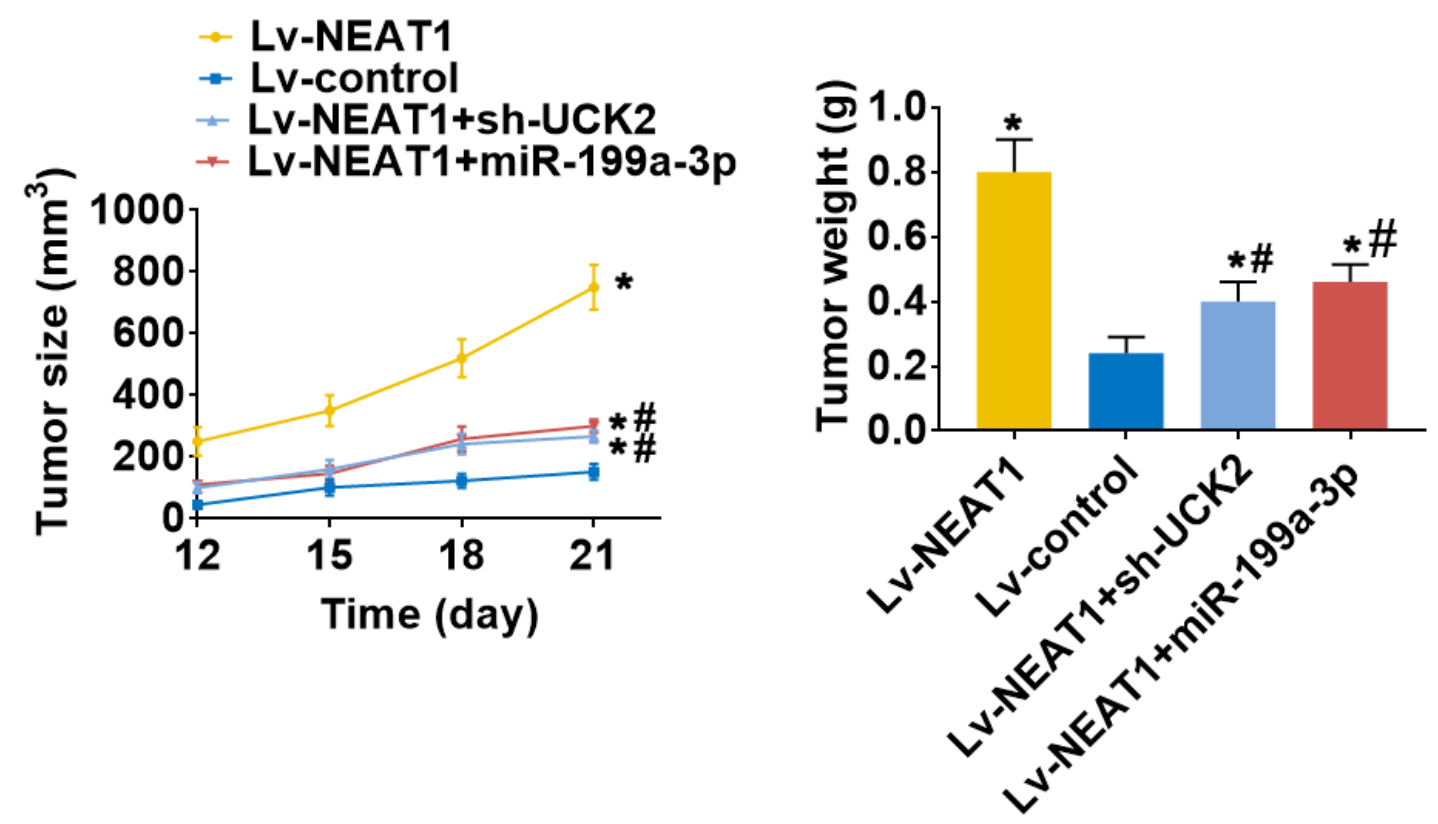

Figure 13

LncRNA-NEAT1 promotes HCC tumor growth through miR-199a-3p/UCK2 in vivo. (a) SNU-182 cells transfected with Lv-NEAT1, Lv-NEAT1 + Lv-miR-199a-3p, or Lv-NEAT1 + Lv-siRNA-UCK2 were subcutaneously injected into nude mice. After 3 weeks, the tumor formation was examined. (b) Tumor 
diameters were measured every 3 days. (c) Tumor weights were measured on day 21 post injection. $* \mathrm{P}<0.05$ compared with transfection negative control. \#P<0.05 compared with Lv-NEAT1.

\section{Supplementary Files}

This is a list of supplementary files associated with this preprint. Click to download.

- Additionalfile1.docx

- Additionalfile1.docx 